\title{
Crack of a helicopter main rotor actuator attachment: failure analysis and lessons learned
}

\section{Rottura di un attacco del servocomando del piatto oscillante di un elicottero: failure analysis e lessons learned}

\author{
L. Allegrucci, F. De Paolis, A. Coletta, M. Bernabei \\ Italian Air Force - Flight Test Centre - Chemistry Department, "De Bernardi" Airport, via Pratica di Mare, 45, 00040 \\ Pomeria (RM) \\ laura.allegrucci@aeronautica.difesa.it
}

\begin{abstract}
A Light Utility Helicopter (LUH), in the course of a training flight, leaving the ground during the taxi to take off, went into an uncontrolled rolling to the right; consequently the helicopter gradually laid down on the right side. The impact with the runway destroyed the rotating blades up to the hubs rotor. The accident investigation focused on main rotor oscillatory plate servo actuators. These components, directly linked to the cloche movements, regulate main rotor blades plane tilt and pitch. Following the preliminary examination, only front servo actuator attachment was found to be broken in two parts. In detail, the present paper deals with the fracture analysis results. The servo actuator attachment material is a 2014 Aluminum alloy extrudate, undergone to T651 heat treatment. Fracture surfaces were examined by optical and electronic microscopy in order to determine the main morphological features and consequently to trace the origin of failure mechanism and causes. The accordance with the specification requirements about alloy composition was verified by quantitative elementary analysis through inductive coupled plasma spectroscopy (ICP); furthermore, semi-quantitative elementary analysis was locally verified by Energy dispersion spectroscopy X ray (EDS_RX). Finally, the hydrogen content of the material was evaluated by the total hydrogen analysis. Microstructural and technological alloy characteristics were verified as well by using metallographic microscopy and hardness testing of the material.

Macroscopic fracture surfaces evidences were characterized by the lack of any significant plastic deformations and by the presence of symmetry compared to the servo actuator axis. Microscopic fracture features of both the investigated surfaces were not coherent to the hypothesis of an impact of the main rotor to the soil. Further achieved evidences, such as grain boundary fracture propagation, the presence of corrosion products, were all in accordance with a Stress Corrosion Cracking (SCC) progressive fracture mechanism.

Finite Element Analysis (FEA) located the highest tensile stress value, when the servo actuator is in its nominal working condition, at the same points where the corrosion products were more concentrated (i.e. in the part of the fracture exposed to oxidative air effect for the longest time). The good agreement between FEA and morphological evidences allowed to determine the progressive fracture origin area, though it was not possible to individuate the crack initiation point. In fact, in correspondence to the initiation area of both the fracture surfaces, shining and flat morphology was found; then there were evidence of plastic deformations, due to the detachment of a servo actuator part.
\end{abstract}


The ICP analysis and hardness testing results were in accordance with the material specification requirements. However, the hydrogen content was one order of magnitude greater than the required value and many and unexpected globular formations were observed on the fracture surface. Part of these were dendritic formations, while the others looked smooth and shining. Further, FESEM boundary grain observation gave evidences of a high presence of precipitates on the investigated surfaces. Hence, observed microstructural characteristics, boundary grain precipitates and globular formations allowed to hypothesize possible overheating/eutectic melting phenomena, occurred during manufacturing processes.

As widely reported in literature, the AA 2014 alloy is one of the aluminum-copper-magnesium-silicon type, employing copper aluminide $(\mathrm{CuAl} 2)$ as the primary precipitation-hardening agent. The need for a maximum $\mathrm{Cu}$ phase dispersion in solid solution requires a heat treatment range with an upper limit $\left(507^{\circ} \mathrm{C}\right)$ that is near to the melting of the eutectics $\left(510^{\circ} \mathrm{C}\right)$. Moreover, since the $1960 \mathrm{~s}$, AA2014 has been defined as sensitive to SCC. This condition is mainly related to the presence of coarse-grained and aligned $\mathrm{CuAl} 2$ precipitates. This arrangement is due to an overheating (more than $507^{\circ} \mathrm{C}$ ) or to a cooling process carried out too slowly.

Microstructural analysis was carried out on three items: 1) a large portion of the broken actuator attachment; 2) on a servoactuator coming from the same production batch; 3) on a servo actuator coming from a different production batch.

The microstructure from the broken actuator attachment showed a great amount of precipitates (second phases) lengthwise aligned to the boundary grain, pores, and also cavities and dendritic globular formations.

Analysis results, morphology evidences and reference images available on scientific literature were found to be in excellent agreement and validated the embrittlement and subsequent SCC mechanism hypotesis (intergranular failure propagation).

In conclusion, flight accident causes are attributable to main rotor actuator attachment failure.

Failure mechanism is classifiable as SCC supported by microstructural anomalies of the material. The investigation of the manufacturing process highlighted how one of the servo actuator batches was not properly produced due to poor control and accuracy of heat treatment temperature and/or cooling time. This led to hydrogen embrittlement and to a microstructural problem (globular formations and boundary grain precipitates). The combination of those phenomena caused an increase of the SCC sensitivity and were the basic progressive failure driving forces.

Nevertheless, as above mentioned, alloy composition was found compliant with the material specification requirements and this just because none of the scheduled quality control tests is able to determine the peculiar microstructural anomalies reported.

SOMMARIO. Un elicottero della classe LUH (Light Utility Helicopter), nel corso di una missione di addestramento, effettuava un decollo rullato e, al momento del distacco da terra, subiva un rollio incontrollabile a destra; negli attimi successivi l'aeromobile si adagiava gradualmente sul fianco destro, con distruzione delle pale fino ai mozzi del rotore per effetto della loro rotazione e interferenza con la pista. L'investigazione è stata focalizzata sui servocomandi del piatto oscillante del rotore principale. Tali comandi regolano l'inclinazione del piano delle pale del rotore principale ed il loro passo in funzione dei comandi di volo. In particolare è risultato che solo uno dei tre attacchi dei servocomandi è stato trovato rotto in due parti. Le evidenze emerse nell'indagine di tale rottura sono oggetto del presente lavoro.

L'attacco del servocomando è costituito da un estruso in lega di alluminio AA 2014 con trattamento termico T651. Le superfici di rottura sono state esaminate mediante microscopia ottica ed elettronica per individuarne le principali caratteristiche morfologiche, al fine di risalire al meccanismo della rottura ed alle sue cause. La rispondenza a specifica della lega è stata effettuata mediante analisi quantitativa elementare ICP (spettroscopia al plasma), analisi semiquantitativa puntuale EDS (spettroscopia a dispersione di energia) ed analisi del contenuto di idrogeno. Le caratteristiche microstrutturali e tecnologiche della lega sono state infine accertate attraverso microscopia metallografica e determinazioni di durezza.

Le evidenze macroscopiche della rottura, caratterizzate dall'assenza di significative deformazioni plastiche e da superfici di rottura simmetriche rispetto all'asse del servocomando, e le evidenze microscopiche rilevate non sono risultate compatibili con l'ipotesi che la rottura sia avvenuta a seguito dell'urto con il terreno da parte del 
rotore principale sul lato destro. Le ulteriori evidenze acquisite sono risultate invece tutte compatibili con un meccanismo di rottura progressiva per tensocorrosione.

L'analisi chimica degli elementi di lega, unitamente alle prove di durezza, hanno confermato l'aderenza del particolare alla specifica, mentre la misura del contenuto di idrogeno ha evidenziato un valore circa un ordine di grandezza superiore a quanto riportato nel certificato di produzione. La presenza sulle superfici di rottura di numerose ed anomale formazioni globulari sia a struttura dendritica che lisce e brillanti ha consentito di ipotizzare che il materiale in fase di produzione abbia subito fenomeni di overheating/eutectic melting che lo ha reso più suscettibile propagazione della corrosione. Tale aspetto è stato confermato attraverso un'estesa analisi microstrutturale e la conduzione di prove di resistenza alla corrosione intergranulare eseguite su porzioni dell'attacco rotto, su uno analogo integro proveniente dallo stesso lotto di produzione, e su campioni di attacchi di lotti diversi. Ciò ha evidenziato per i primi una maggiore stratificazione dei precipitati (seconde fasi) lungo i bordi longitudinali dei grani, oltre a pori, cavità e precipitati globulari a struttura dendritica correlabili ad una maggiore suscettibilità alla corrosione.

Le evidenze acquisite hanno consentito di ricondurre la causa della rottura ad un fenomeno di tensocorrosione favorito da un'anomalia del materiale di cui l'attacco è costituito. Tale anomalia è avvenuta nel processo di produzione del lotto a cui l'attacco appartiene per eventi di non controllo del surriscaldamento o del raffreddamento. Ciò ha causato sia l'eccessivo contenuto di idrogeno sia le alterazioni microstrutturali che hanno aumentato la suscettibilità del materiale alla corrosione intergranulare e che hanno costituito le driving forces del meccanismo di rottura osservata.

KEYwords. Helicopter; Failure Analysis; Stress Corrosion; Alluminum; Microstructure.

\section{INTRODUZIONE: INCIVOLO E MODALITÀ DI CONDUZIONE DELL'INDAGINE TECNICA}

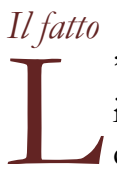

incidente è avvenuto durante un decollo rullato in missione di addestramento. Il rollio incontrollabile ha costretto il velivolo ad adagiarsi sul fianco destro con l'effetto della distruzione delle pale per interferenza con la pista. La dinamica dell'incidente ha suggerito ai tecnici impegnati nell'investigazione di focalizzare l'attenzione sui servocomandi del piatto oscillante del rotore principale (Fig. 1). La funzione dei servocomandi è quella di regolare la posizione del piatto oscillante fisso del rotore principale e conseguentemente di determinare l'inclinazione del piano delle pale ed il loro passo in funzione dei comandi di volo.

Dall'osservazione in loco è risultato rotto uno dei tre attacchi dei servocomandi.

Gli attacchi sono stati sottoposti ad ulteriori indagini con lo scopo di determinare un eventuale nesso causale tra la rottura osservata e l'incivolo.

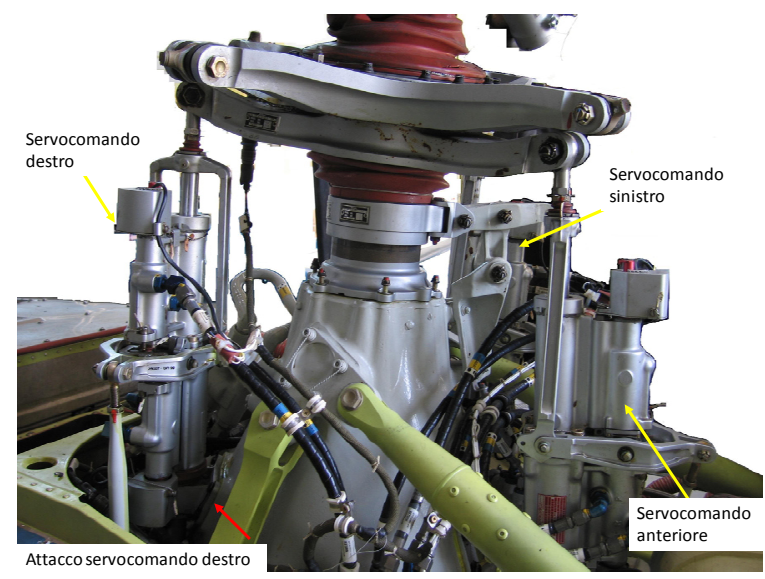

Figura 1: Collocazione dei servocomandi sul rotore.

Figure 1: Position of the main rotor actuators. 


\section{I reperti esaminati}

Sono stati esaminati $\mathrm{i}$ tre attacchi dei servocomandi del velivolo incidentato, tutti provenienti dallo stesso lotto di produzione, ed altri due attacchi nuovi, provenienti da altri due lotti di produzione, come riportato in Tab. 1. L'attacco rotto è stato studiato al fine di individuare le caratteristiche ed il meccanismo della rottura, gli altri quattro particolari sono stati studiati per confronto al fine di definire ed approfondire le cause che hanno originato la rottura.

\begin{tabular}{cccc}
\hline $\begin{array}{c}\text { Denominazione attacco } \\
\text { servocomando }\end{array}$ & Posizione & $\begin{array}{c}\text { Lotto di } \\
\text { produzione }\end{array}$ & Note \\
$\mathrm{A}$ & anteriore & $\mathrm{X}$ & rotto \\
$\mathrm{S}$ & sinistro & $\mathrm{X}$ & integro \\
$\mathrm{D}$ & destro & $\mathrm{X}$ & integro \\
1 & $\mathrm{~N} / \mathrm{A}$ & $\mathrm{Y}$ & nuovo \\
2 & $\mathrm{~N} / \mathrm{A}$ & $\mathrm{Z}$ & nuovo \\
\hline
\end{tabular}

Tabella 1: Descrizione degli attacchi dei servocomandi esaminati. Table: Details of the examined actuator attachments.

Gli attacchi fissano i servocomandi alle strutture del rotore principale in posizione anteriore, destra e sinistra, come evidenziato dallo schema in Fig. 2 e in Fig. 3. Ciascun attacco è vincolato con 4 bulloni come in Fig. 4.

L'ispezione ha rilevato la rottura dell'attacco anteriore A in due parti, Fig. 5;

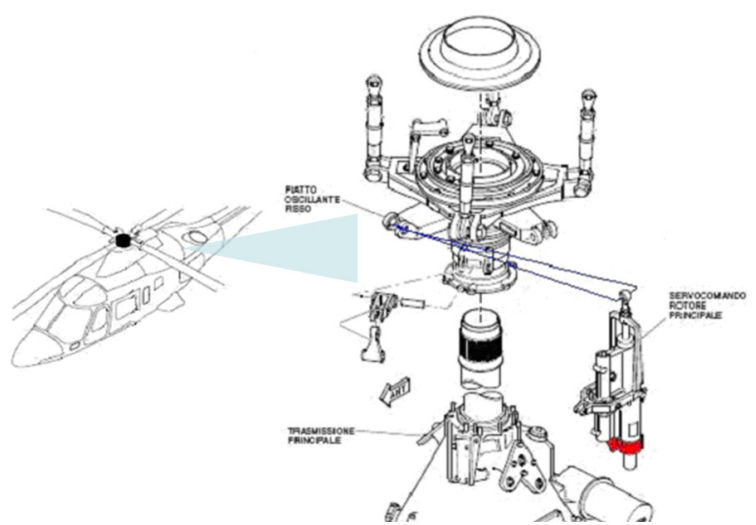

Figura 2: Schema della collocazione del particolare rotto Figure 2: Sketch of the failed actuator attachment location

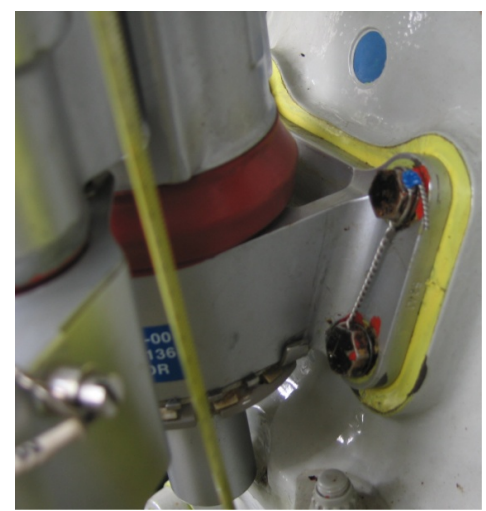

Figura 4: Attacco integro in condizioni di servizio. Figure 4: Intact attachment in exercise condition.

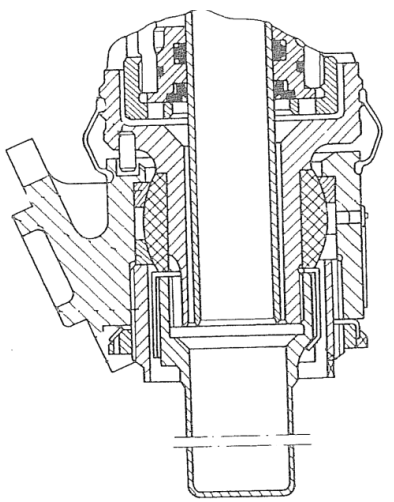

Figura 3: Schema attacco e servocomando assemblati Figure 3: Drawing of assembled actuator and actuator attachment.

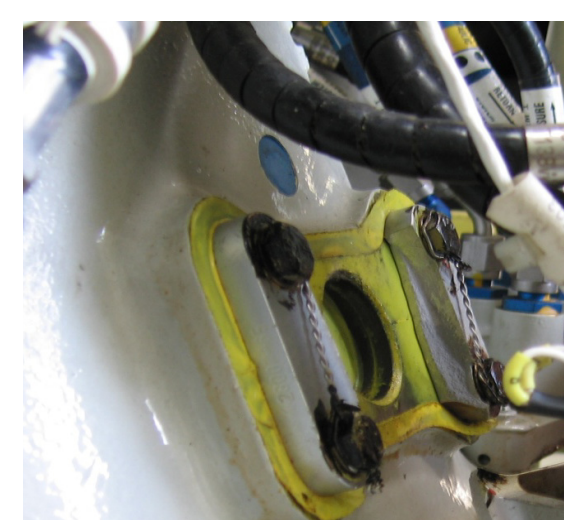

Figura 5: Attacco rotto. Figure 5: Broken attachment.

Tutti e tre i frammenti rinvenuti sono stati esaminati: un frammento principale (1 in Fig. 6), rinvenuto ancora associato al servocomando, e due altri frammenti ( 2 e 3 in Fig. 6), rinvenuti ancora vincolati alla struttura del rotore principale dai 
rispettivi bulloni. Rispetto alla posizione originaria il frammento principale è stato trovato abbassato di $8 \mathrm{~cm}$ e spostato in avanti di $8 \mathrm{~cm}$, ma non spostato a destra o sinistra.

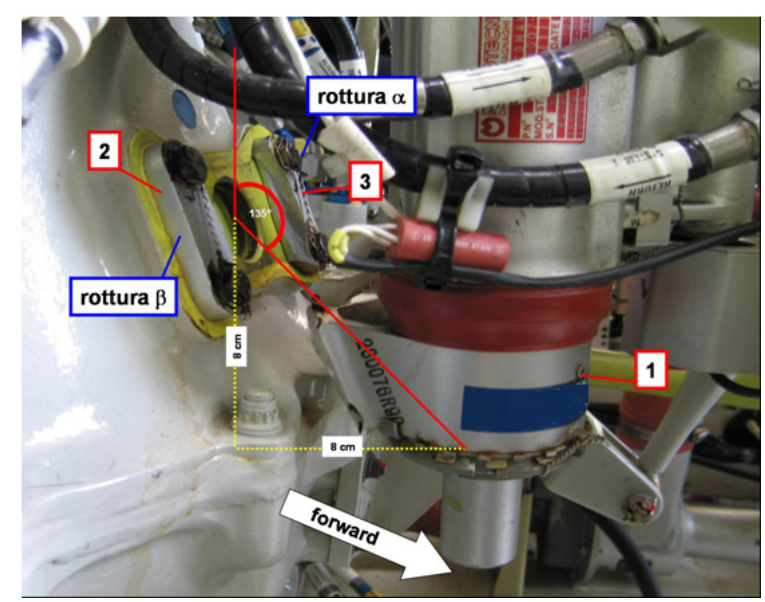

Figura 6: Distacco dell'attacco, come rinvenuto.

Figure 6: Actuator displacement as observed after failure.

\section{Il protocollo di indagine}

Sul reperto rotto è stato seguito un protocollo di indagine che ha consentito di esaminare e documentare gli aspetti macrofrattografici e microfrattografici. Su tutti gli attacchi sono stati effettuati gli esami metallografici, le misure di durezza ed anche le analisi chimiche quantitative elementari, semiquantitative puntuali, determinazione del contenuto di idrogeno, prove di suscettibilità alla corrosione intergranulare per verificare la rispondenza a specifica del materiale in termini chimico-fisici e tecnologici e per porre a confronto il reperto rotto con altri particolari analoghi dello stesso lotto di produzione e di lotti di produzione differenti. Infine, in base alle rilevanze ottenute ed ai dati disponibili, è stato possibile costruire un modello numerico per stimare i carichi applicati e per individuare il punto maggiormente sollecitato.

\section{Apparati e strumentazione}

Gli esami macrofrattografici sono stati condotti con microscopio ottico Leica M205C. Gli esami microfrattografici sono stati effettuati mediante microscopio elettronico FESEM-Zeiss Leo-Supra 35 equipaggiato con microanalizzatore di fluorescenza dei raggi X a dispersione di energia (EDS) Oxford INCA MICS. Gli esami metallografici sono stati condotti con microscopio metallografico Leica DM 6000. Le prove di durezza sono state effettuate secondo il metodo Rockwell T con un carico di $30 \mathrm{~kg}$ e penetratore sferico di acciaio $\varnothing 1 / 16 "$. Le analisi chimiche elementari sono state effettuate mediante ICP ottico Perkin Elmer Optima 2100 DV. La misura del contenuto di idrogeno, basata sulla rilevazione della variazione di conducibilità termica del gas inerte con cui è stata effettuata l'estrazione termica dell'idrogeno dai campioni, è stata effettuata mediante Analizzatore LECO RH-402.

\section{RISULTATI}

\section{Esame visivo e macrofrattografico}

T e due rotture rinvenute sull'attacco sono definite $\operatorname{con} \alpha$ (lato sinistro dell'elicottero) e $\beta$ (lato destro dell'elicottero) e schematizzate nel disegno a destra, Fig. 7.

Le superfici (A1 e A2 superfici complementari della rottura $\alpha$, B1 e B2 superfici complementari della rottura $\beta$ ) mostrano evidenti analogie, Fig. 8 e Fig. 9: tre tipi di propagazione (denominate tipo 1, tipo 2, tipo 3) con identiche morfologie, collocazioni ed inclinazioni e con estensioni paragonabili, come riportato nel confronto in Tab. 2. In entrambi i casi non si osservano deformazioni plastiche associate e sono invece presenti segni di spalmature dirette dall'alto verso il basso. Quanto osservato indica una simmetria assiale nel meccanismo di rottura, presumibilmente diretta lungo la direzione coda-prua del velivolo, senza componenti destra o sinistra, con i segni di battitura associabili all'urto tra superfici accaduto per effetto dell'uscita dalla sede del frammento centrale in accordo con la posizione finale nella quale è stato rinvenuto il frammento principale (angolo di $135^{\circ}$ rispetto alla normale al piano dell'elicottero). 


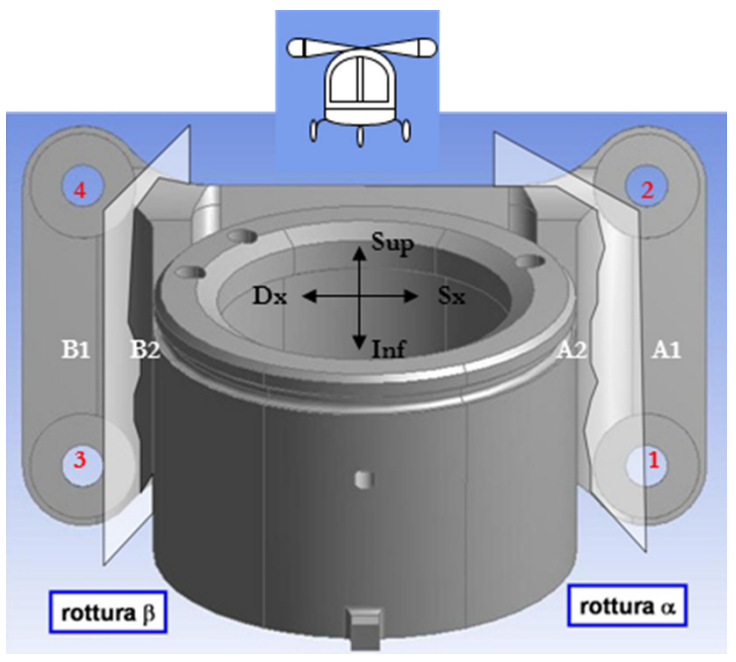

Figura 7: Schema delle rotture rinvenute sull'attacco A. Figure 7: Scketch of the cracks found on the attachment A.
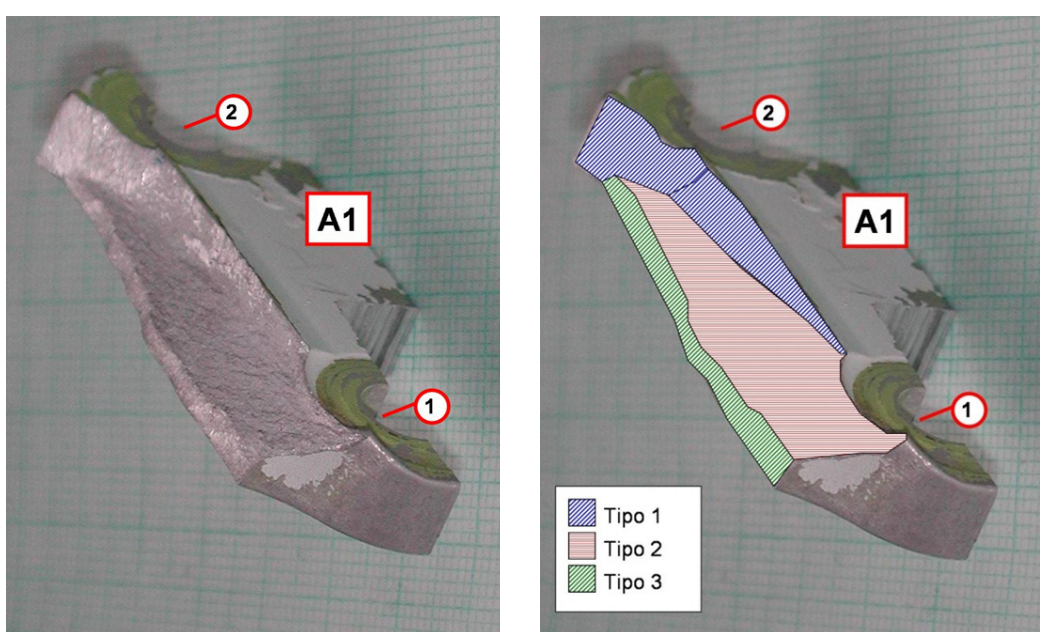

Figura 8: Rottura A1 e differenti tipi di propagazione.

Figure 8: A1 crack surface with its different propagation areas (type 1-3).

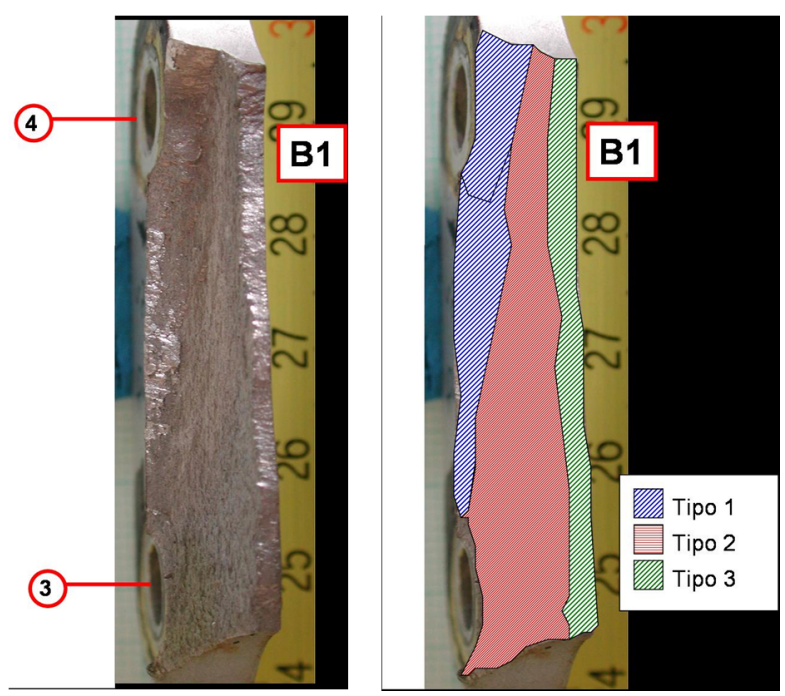

Figura 9: Rottura B1 e differenti tipi di propagazione.

Figure 9: B1 crack surface with its different propagation areas(type 1-3). 


\begin{tabular}{|c|c|c|c|}
\hline Tipo & Caratteristiche morfologiche & Estensione su A1 & Estensione su B1 \\
\hline 1 & $\begin{array}{l}\text { planare, costituita da due zone a } \\
\text { diversa inclinazione rispetto alla } \\
\text { normale terra-cielo ma entrambe } \\
\text { perpendicolari al piano di giacenza dei } \\
\text { fori }\end{array}$ & $\begin{array}{l}39 \% \text {. Si estende dal bordo } \\
\text { superiore }- \text { assottigliandosi - } \\
\text { fino alla sede del dado in foro } \\
1\end{array}$ & $\begin{array}{l}40 \% \text {. Si estende dal bordo superiore, } \\
\text { assottigliandosi, in direzione della } \\
\text { sede del foro } 3 \text {, arrestandosi prima }\end{array}$ \\
\hline 2 & $\begin{array}{l}\text { non planare e distribuita su piani con } \\
\text { prevalente componente parallela al } \\
\text { piano di giacenza dei fori } \\
\text { planare e perpendicolare alla superficie } \\
\text { che costituisce la piastra posteriore } \\
\text { dell'attacco, è parallela alla normale } \\
\text { terra-cielo }\end{array}$ & $\begin{array}{l}38 \% \text {. Si estende dai bordi della } \\
\text { superficie di tipo } 1 \text { fino all'area } \\
\text { sottostante il foro } 1 \\
23 \% \text {. Ha altezza media pari a } \\
1,5 \mathrm{~mm}\end{array}$ & $\begin{array}{l}42 \% \text {. Si estende dai bordi della } \\
\text { superficie di tipo } 1 \text { fino all'area } \\
\text { sottostante il foro } 4 \\
18 \% \text {. Ha altezza media pari a } 1,5 \mathrm{~mm}\end{array}$ \\
\hline
\end{tabular}

Tabella 2: Caratteristiche morfologiche delle 3 aree individuate sulle rotture A1 e B1.

Table 2: Morphological characteristics of the 3 areas observed on A1 and B1 crack surfaces.

\section{Esame microfrattografico}

Gli esami al microscopio ottico ed elettronico hanno confermato le analogie di progressione delle due rotture.

In particolare, sui differenti tipi di propagazione identificati, sono state effettuate osservazioni al microscopio elettronico nei punti indicati con le lettere a-h in Fig. 10.

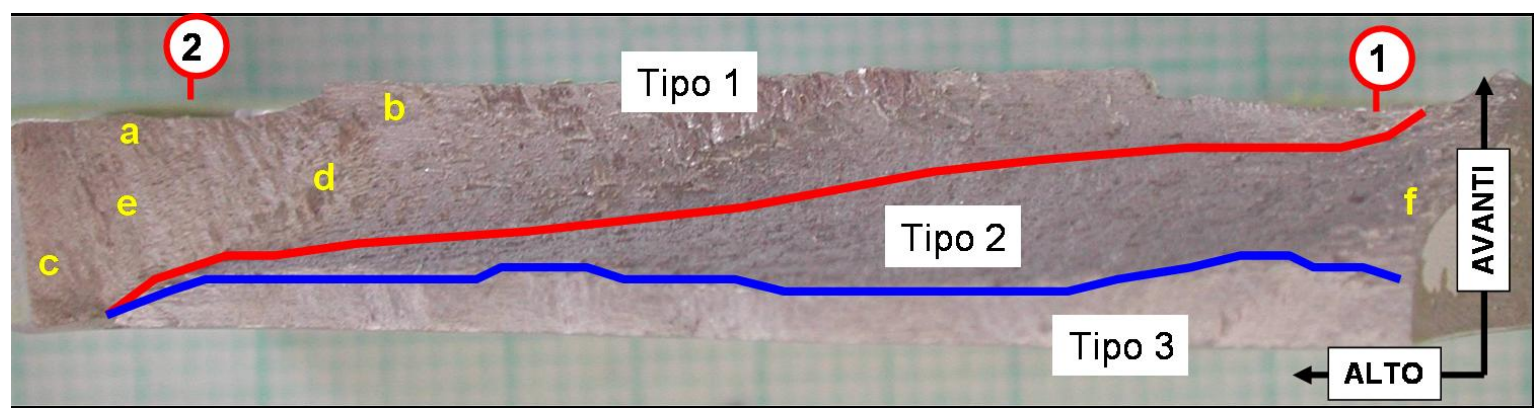

Figura 10: Superficie di rottura particolare A1.

Figure 10: Details of the A1 crack surface.

In dettaglio, nel punto a posto nella parte immediatamente sottostante il foro 2, si osservano segni di spalmatura meccanica della superficie, e, nell'intorno di tale punto, è visibile una fascia di profondità pari a circa $250 \mu \mathrm{m}$ che presenta maggiore densità dei prodotti di corrosione, macchie chiare e scure in Fig. 11.

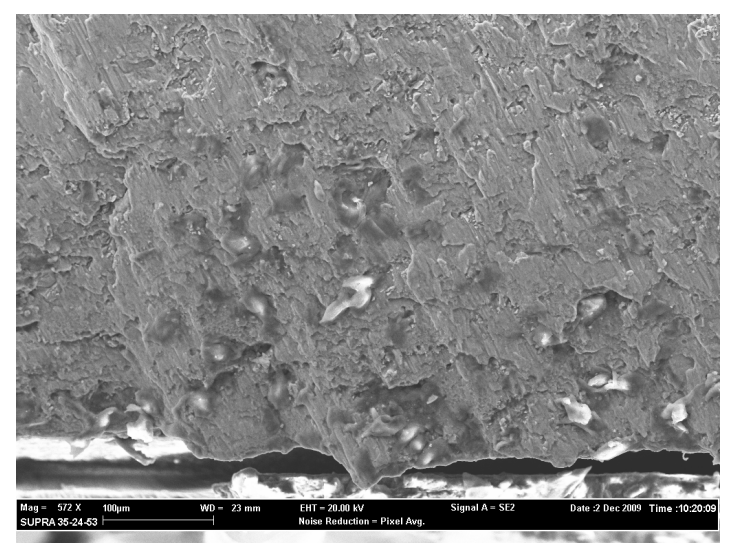

Figura 11: Superficie A1, punto a. Figure 11: A1 surface, point a.

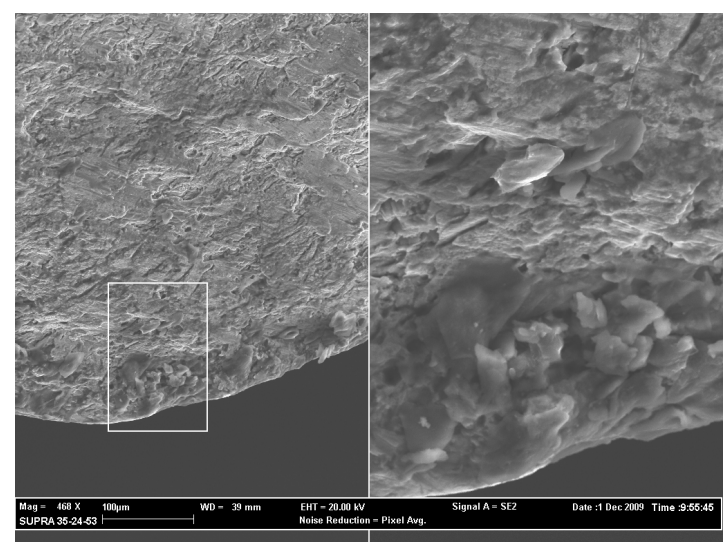

Figura 12: A1 superficie di rottura, punto c. Figure 12: A1 surface, point c. 
Nel punto c (Fig. 12), posizionato in prossimità del bordo superiore dell'attacco e vicino alla sua superficie posteriore, si osserva la presenza significativa di prodotti di corrosione.

In entrambi i casi:

- la superficie di tipo 1 è caratterizzata dalla presenza di prodotti di corrosione, soprattutto nella zona in prossimità del foro 2 , e da una morfologia a carattere intergranulare, a grani orientati con la presenza, rilevabile solo ad elevato ingrandimento, di micropori localizzati sui bordi dei grani, Fig. 13 e Fig. 14;

- la superficie di tipo 2 è caratterizzata dalla stessa morfologia della propagazione di tipo 1, con accentuata orientazione dei grani, Fig. 15;

- la superficie di tipo 3 è caratterizzata da deformazioni da strappo in direzione della superficie di tipo 2 ed un'accentuata e coerente orientazione dei micropori di bordo grano, Fig. 16;

Su tutta la superficie si rinvengono molteplici particelle globulari, anche incluse nella matrice metallica, alcune lisce e brillanti (cerchio blu), altre a struttura dendritica (cerchio rosso) Fig. 17, Fig. 18 e Fig. 19.

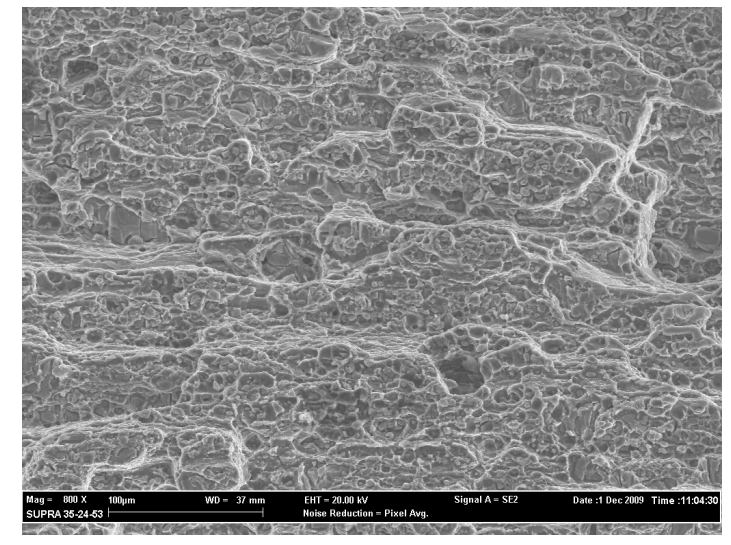

Figura 13: Superficie A1 tipo 1.

Figure 13: A1 surface, type 1.

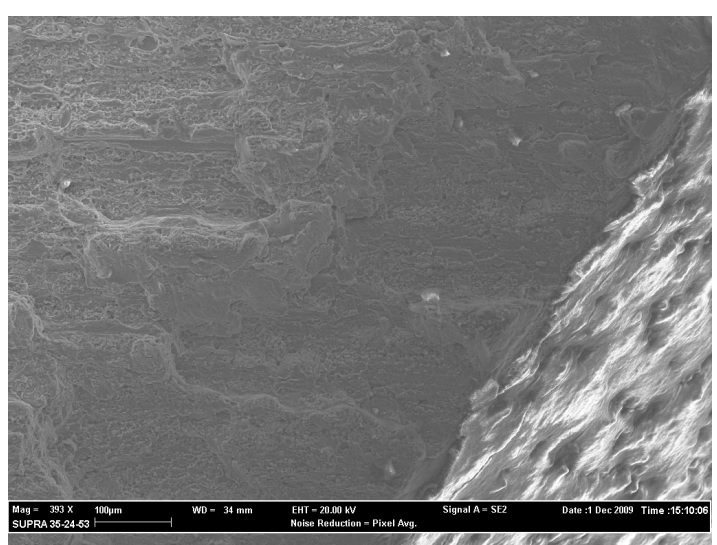

Figura 15: Superficie A1 tipo 2.

Figure 15: A1 surface, type 2.

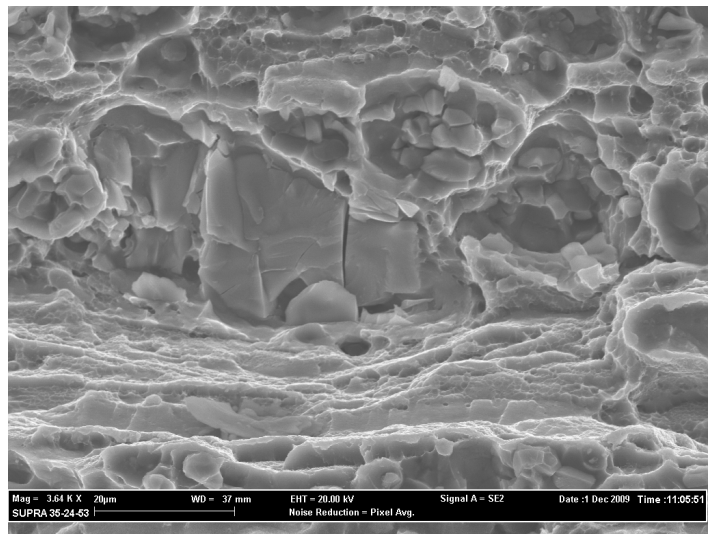

Figura 14: Superficie A1 tipo 1 Figure 14: A1 surface, type 1.

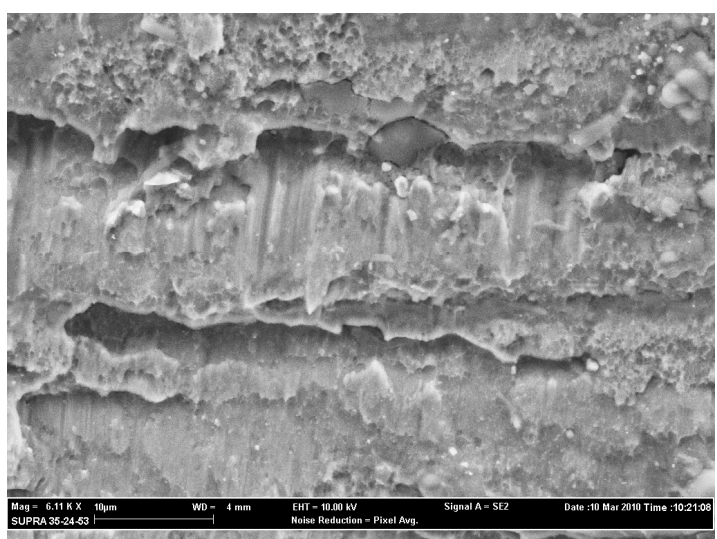

Figura 16: Superficie A1 tipo 3

Figure 16: A1 surface, type 3.

Le superfici di rottura esaminate presentano carattere intergranulare fragile.

L'orientazione dei micropori nelle superfici di tipo 3 indica che queste sono state interessate da un carico tensionale con componente ortogonale alla parte dell'attacco costituente la piastra di fissaggio; tale orientazione è coerente con un distacco del frammento centrale dell'attacco per avanzamento lungo la direttrice prua-coda del velivolo.

Le superfici di rottura, nelle zone di propagazione di tipo 1, presentano una maggiore quantità di prodotti corrosivi ad indicare una possibile preesistenza di questa zona rispetto alle altre due.

Su tutte le superfici si rinvengono numerose ed anomale formazioni globulari sia a struttura dendritica che lisce e brillanti. 


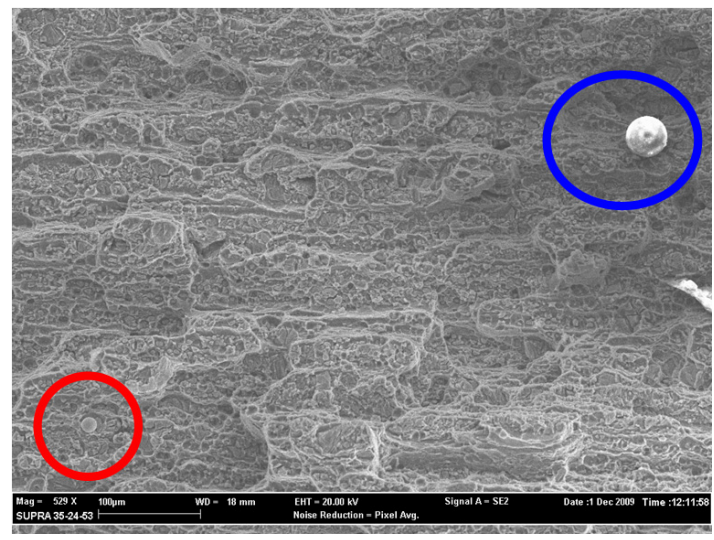

Figura 17: Particelle globulari.

Figure 17: Globular formations.

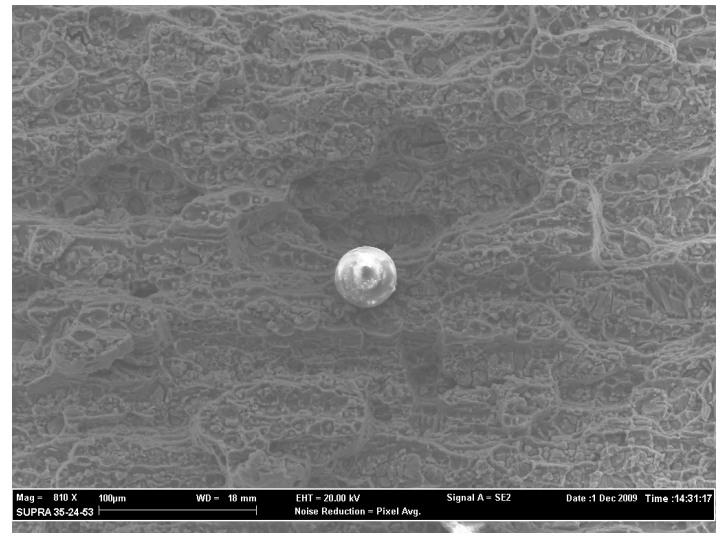

Figura 18: Particelle globulari iridescenti. Figure 18: Iridescent globular formations.

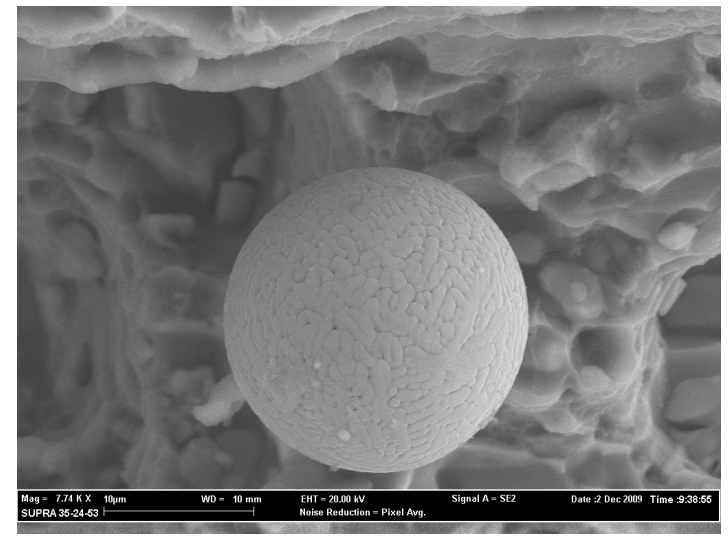

Figura 19: Particelle globulari dendritiche. Figure 19: Dendritic globular formations.

\section{Analisi chimica elementare}

L'analisi quantitativa elementare del materiale costituente l'attacco è stata eseguita mediante spettroscopia al plasma ICP OES fornendo i risultati riportati in Tab. 3.

\begin{tabular}{cccccccccc}
\hline & \multicolumn{10}{c}{ Elementi } & & & & \\
& $\mathrm{Al}$ & $\mathrm{Cu}$ & $\mathrm{Mn}$ & $\mathrm{Si}$ & $\mathrm{Mg}$ & $\mathrm{Fe}$ & $\mathrm{Cr}$ & $\mathrm{V}$ & $\mathrm{Zn}$ \\
\hline \% in peso & base & 4.56 & 0.72 & 0.70 & 0.71 & 0.19 & 0.05 & 0.05 & 0.05 \\
\hline
\end{tabular}

Tabella 3: Composizione chimica del materiale costituente l'attacco. Table 3: Chemical composition of the attachment alloy.

La composizione elementare è riconducibile alla lega leggera a base Alluminio del tipo AA2014, in accordo alle specifiche trasmesse per il componente investigato.

\section{Misura del contenuto di idrogeno}

L'intergranularità ed il carattere fragile della morfologia della rottura hanno indirizzato l'indagine tecnica verso la verifica del contenuto di idrogeno nel materiale costituente gli attacchi. Sono stati selezionati per l'analisi campioni prelevati dall'attacco rotto e da due attacchi integri di cui uno dello stesso lotto e montato sullo stesso elicottero ed uno appartenente ad un diverso lotto di produzione.

I risultati ottenuti sono riportati nella Tab. 4 e sono confrontati con i due valori misurati dalla ditta produttrice. 


\begin{tabular}{cccc}
\hline $\begin{array}{c}\text { Denominazione attacco } \\
\text { servocomando }\end{array}$ & $\begin{array}{c}\text { Lotto di } \\
\text { produzione }\end{array}$ & Misurato & Da Report \\
A & X & 1.02 & 0.09 \\
D & X & 0.33 & 0.09 \\
1 & Y & 0.34 & incognito \\
\hline
\end{tabular}

Tabella 4: Contenuto di idrogeno in attacchi provenienti da lotti diversi.

Table 4: Hydrogen content of attachments derived from different batches.

I valori misurati non concordano con quelli forniti, ma sono superiori. In particolare nel caso dell'attacco A tali valori sono di un ordine di grandezza superiore, ma sono anche tre volte superiori agli altri due attacchi analizzati.

\section{Esame micro strutturale: superficie di rottura}

L'esame metallografico è stato effettuato su una sezione del frammento contenente la superficie di frattura B1, a circa 1,5 $\mathrm{cm}$ dal foro 4.

Sulle superfici di tipo 1 e tipo 2 si osserva che la rottura propaga in modo intergranulare, Fig. 20; si rinvengono inoltre cavità a morfologia dendritica, Fig. 21.

Diversamente sulla superficie di tipo 3 si riscontra che la rottura non segue il bordo dei grani ma definisce un profilo lineare, Fig. 22; quest'ultima è compatibile con fenomeni di battitura superficiale o con meccanismi di propagazione transgranulare, coerente con il carico tensionale e le deformazioni da strappo già evidenziati nel corso degli esami microfrattografici.

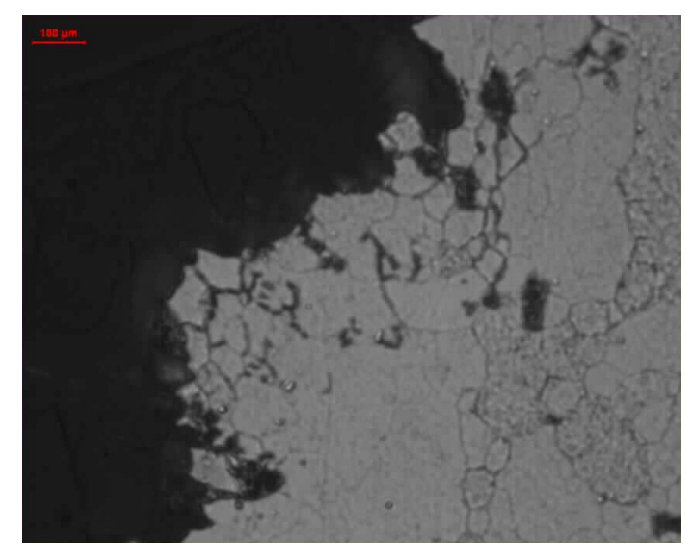

Figura 20: Intergranularità della superficie di tipo 1. Figure 20: Intergranular propagation on type 1 surface.

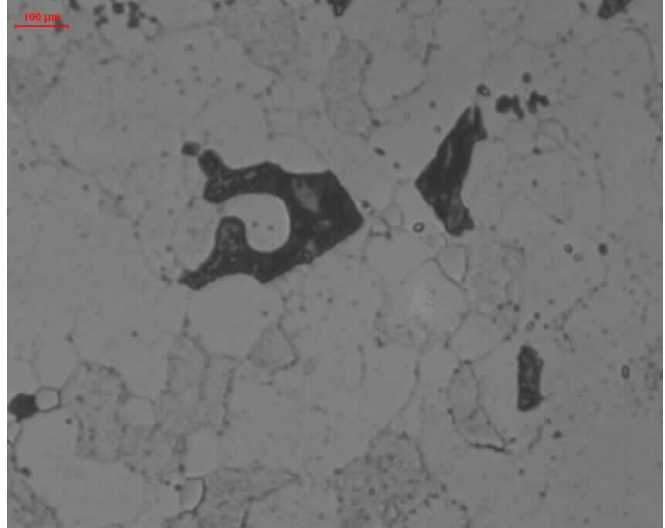

Figura 21: Cavità a morfologia dendritica. Figure 21: Dendritic cavities.

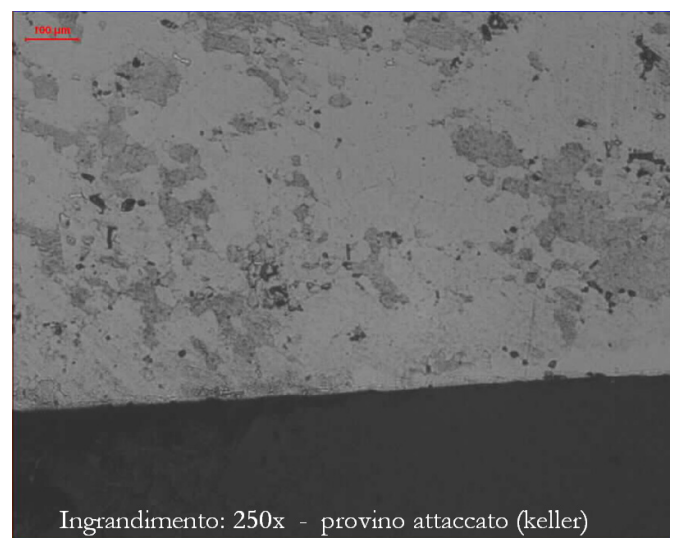

Figura 22: Morfologia transgranulare nella propagazione di tipo 3.

Figure 22: Transgranular morphology as observed on type 3 propagation. 
Esame micro strutturale: confronto tra gli attacchi

Le anomalie emerse dalla misura del contenuto di idrogeno hanno reso indispensabile un confronto microstrutturale tra l'attacco A, l'attacco D e gli attacchi 1 e 2.

L'esame è stato effettuato su sezioni trasversali rispetto al verso di laminazione, che per progetto è parallelo alla parte dell'attacco che agisce quale piastra di vincolo per mezzo dei quattro bulloni.

Le sezioni sono state effettuate su provini prelevati in due punti diversi per l'Attacco A: un provino è stato prelevato nei pressi del foro inferiore, cerchio verde in Fig. 23, mentre l'altro è stato prelevato dalla zona centrale dell'attacco, cerchio rosso in Fig. 21, mentre per gli altri tre attacchi è stata effettuata la sola sezione nella zona centrale.
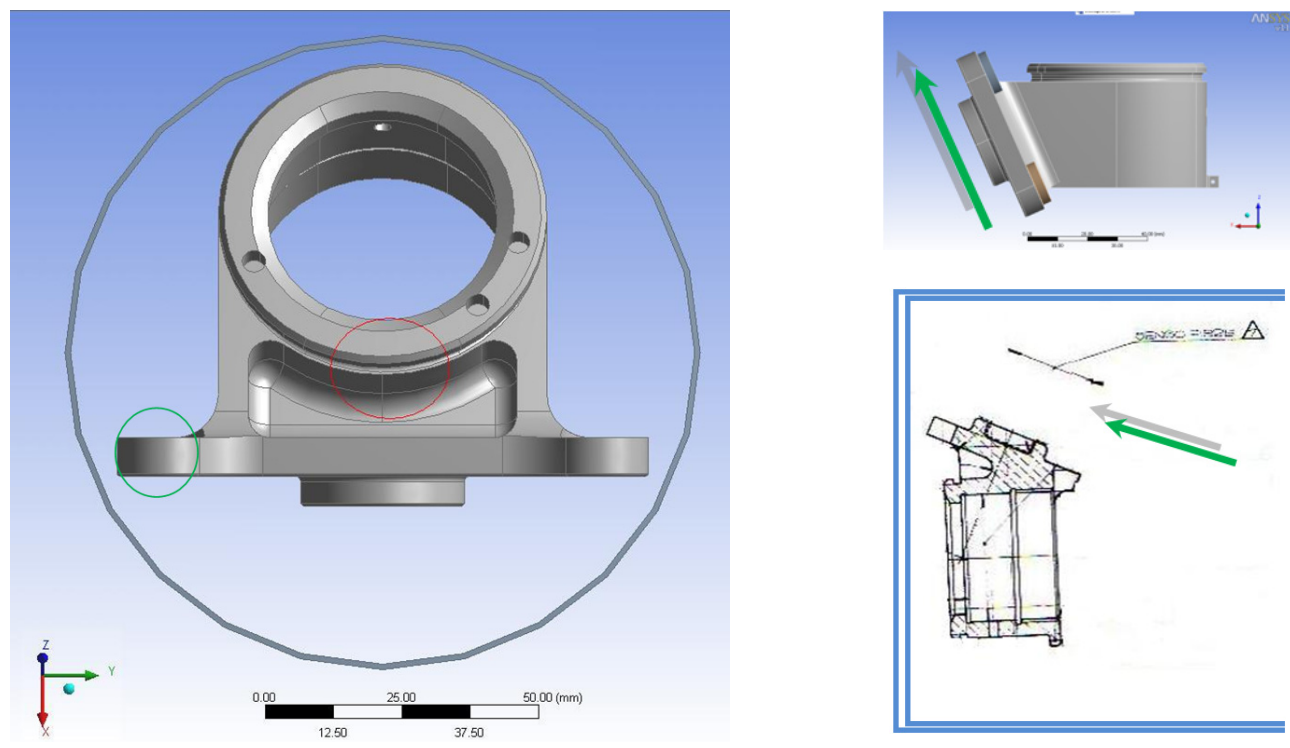

Figura 23: Localizzazione dei provini per microstruttura (Freccia verde: senso di laminazione). Figure 23: Position of specimens for microstructural analysis (Green arrow: rolling direction).

\section{Attacco A}

Su entrambi i provini, si rinviene stratificazione delle seconde fasi - di colore grigio chiaro, grigio scuro e nero - lungo i bordi longitudinali dei grani, orientati come da specifica di produzione, Fig. 24.

Si rinvengono tra l'altro strutture circolari, Fig. 25, compatibili con le strutture dendritiche già individuate sulla superficie di rottura A1 mediante microscopia elettronica (Fig. 18 e Fig. 19).

Su tale provino si osservano anche pori e cavità, compatibili con possibile asportazione di precipitati globulari e con porosità da gas, come evidente anche dalla comparazione con l'atlante di metallografia, Fig. 26 [1].

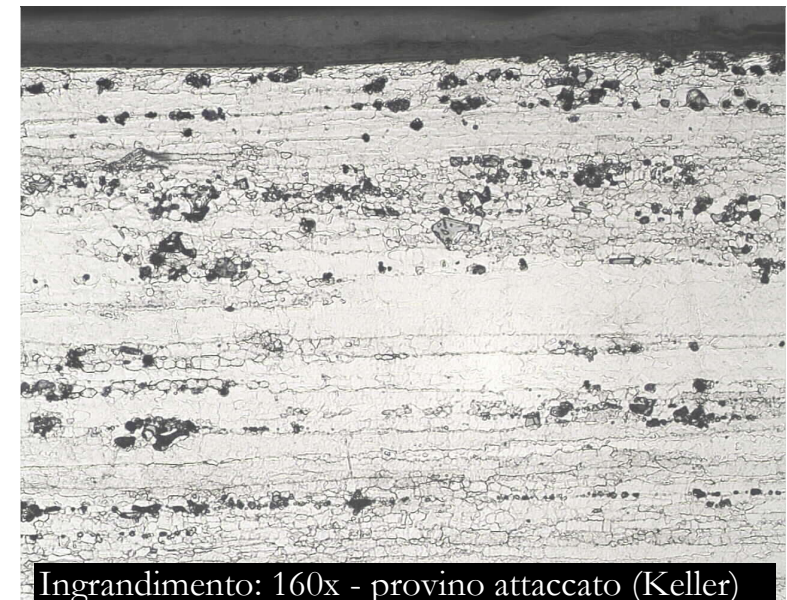

Figura 24: Sezione accanto al foro. Figure 24: Section near the hole.

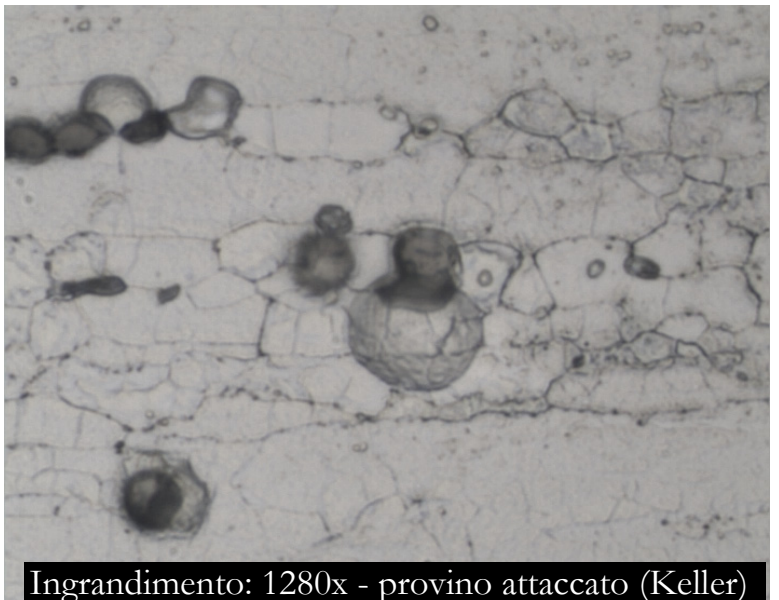

Figura 25: Sezione accanto al foro. Figure 25: Section near the hole. 

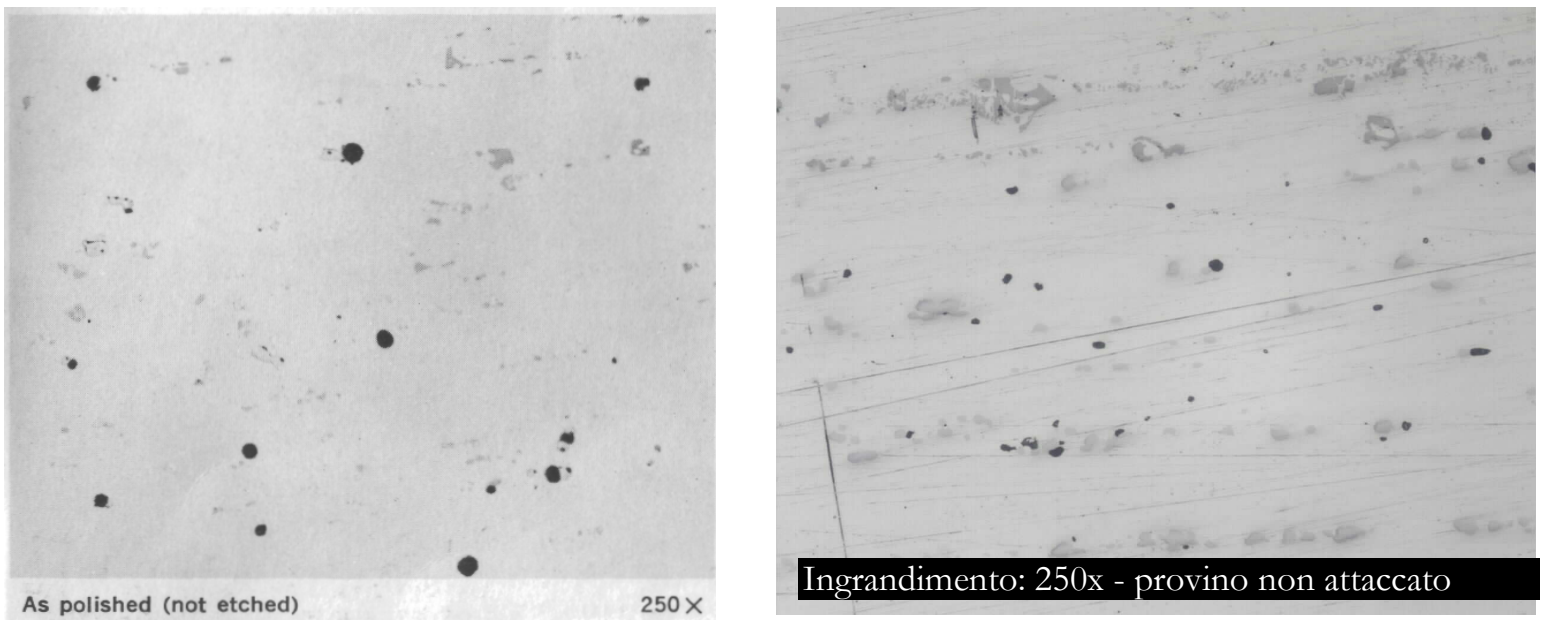

Figura 26: Confronto tra immagine del provino accanto al foro e manuale di riferimento.

Figure 26: Comparison between images from Atlas of Metallography (left) and the investigated specimen (right).

Sul provino prelevato dalla zona centrale dell'attacco le porosità osservate sembrano maggiormente presenti, come si può vedere in luce polarizzata, Fig. 27 immagine A. Anche sulla superficie esterna la stratificazione comporta disomogeneità di composizione, immagine B in Fig. 27.
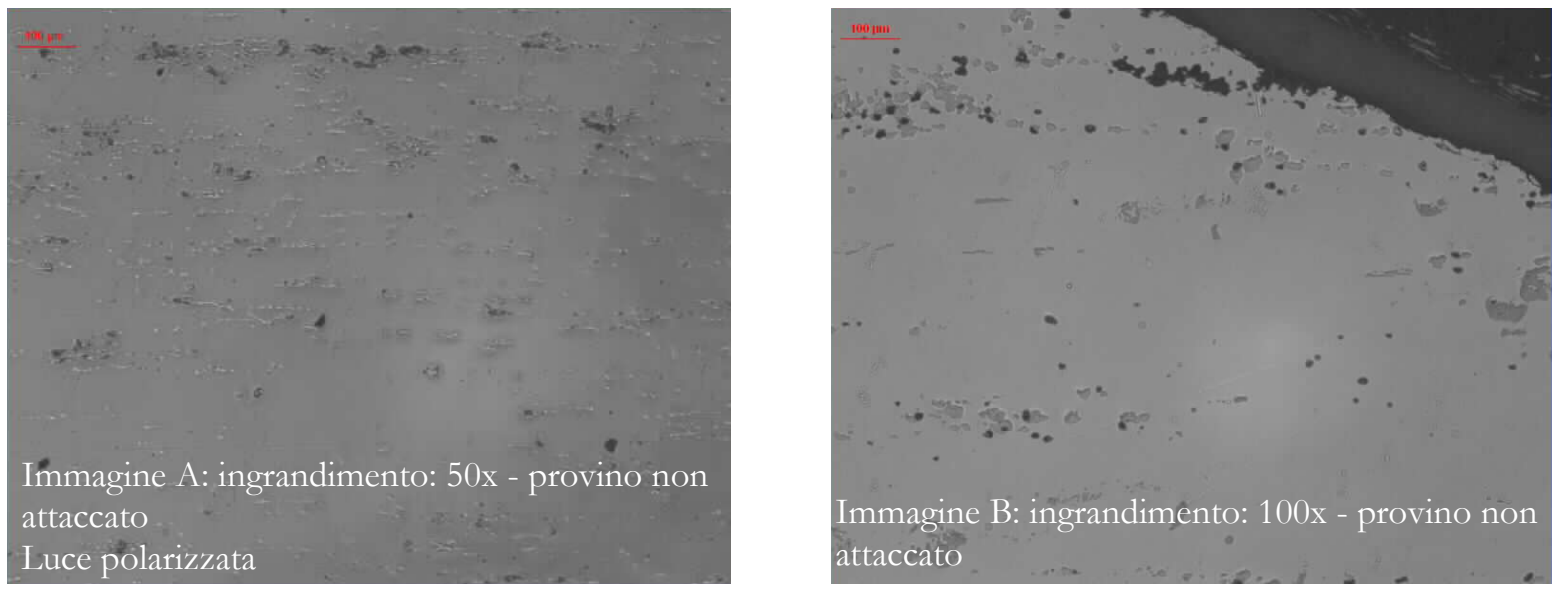

Figura 27: Porosità provino centrale.

Figure 27: Porosity of the specimen obtained from the attachment central area.

\section{$\underline{\text { Attacco D }}$}

Anche in questo caso si rinviene stratificazione delle seconde fasi - di colore grigio chiaro, grigio scuro e nero - lungo i bordi longitudinali dei grani, orientati come da specifica di produzione, nonché pori e cavità, compatibili con possibile asportazione di precipitati globulari e con porosità da gas, Fig. 28.

Tra le altre, si rinviene una struttura circolare di grande diametro (circa $140 \mu \mathrm{m}$ ), di aspetto compatibile con le strutture dendritiche già individuate sulla superficie di rottura A1 mediante microscopia elettronica, Fig. 29.

\section{Attacchi 1 e 2}

Su entrambi i provini (provenienti da lotti di produzione differenti) si rinviene una microstruttura diversa da quanto osservato sui provini ottenuti dagli attacchi A (rotto) e D (integro) dello stesso lotto e montati sull'elicottero oggetto di indagine, Fig. 30 e Fig. 31.

La stratificazione delle seconde fasi di colore grigio chiaro è meno pronunciata, mentre le fasi più scure sono molto più fini. Si rinvengono altresì rare e piccole cavità. 


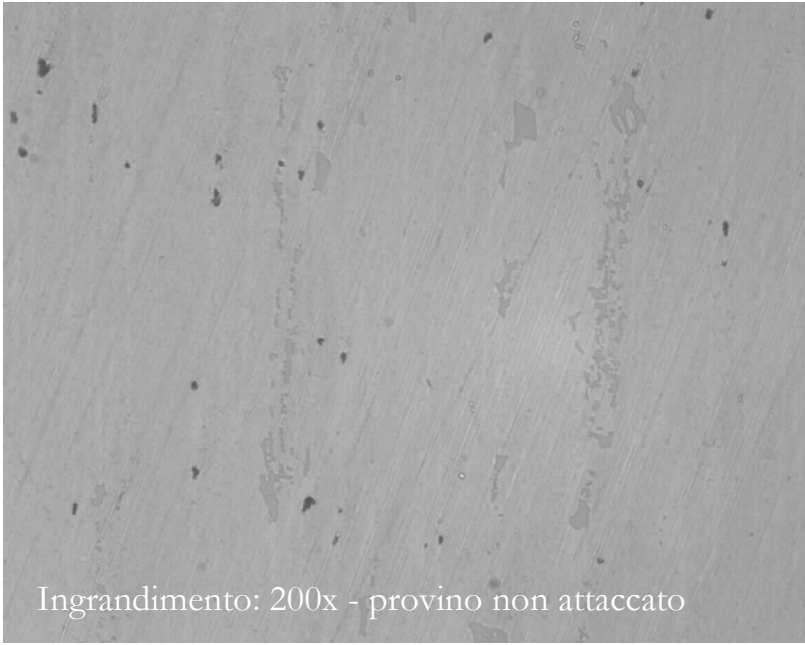

Figura 28: Porosità provino centrale attacco D. Figure 28: Attachment D, central specimen porosity.

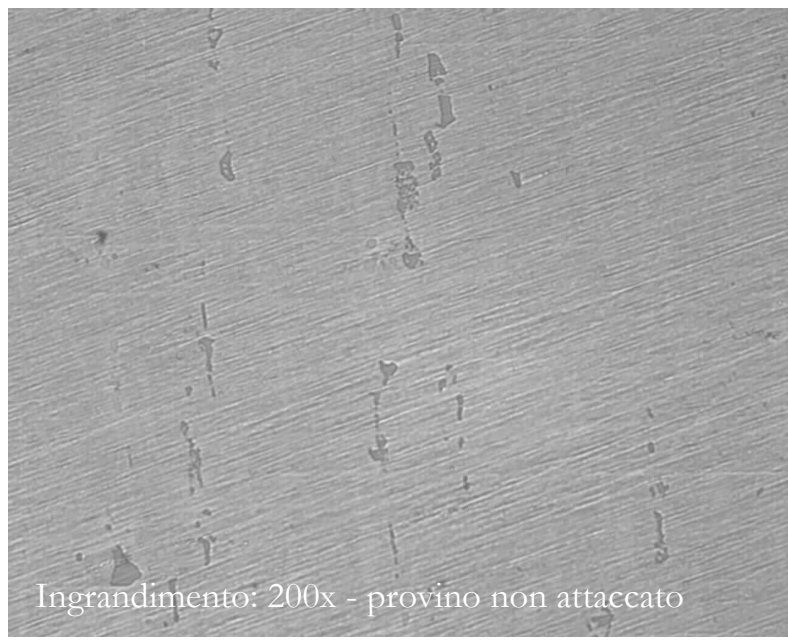

Figura 30: Provino centrale attacco 1.

Figure 30: Central specimen, attachment 1.

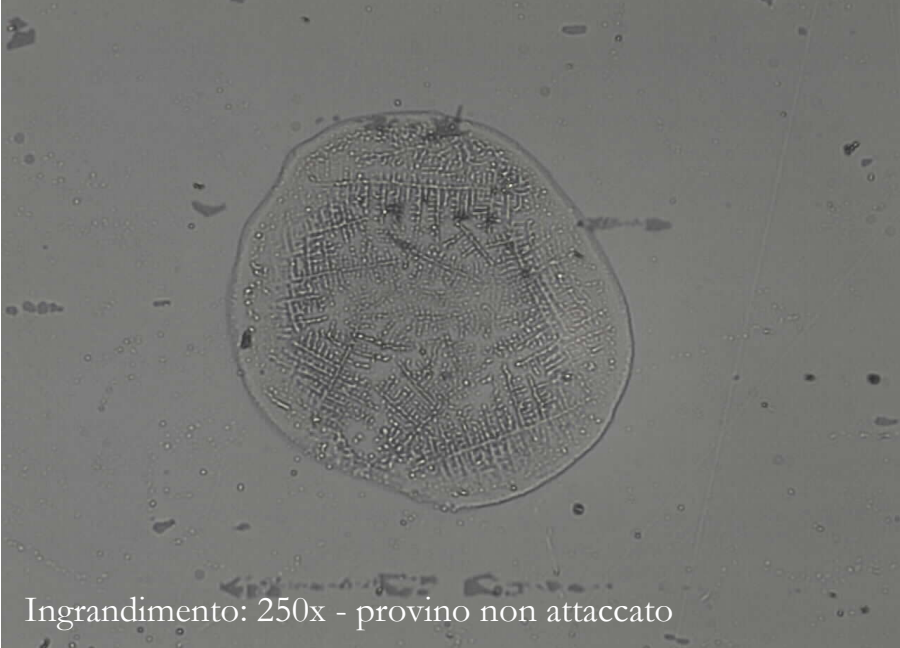

Figura 29: Struttura dendritica provino centrale attacco D.

Figure 29: Attachment D, central specimen dendritic structure.

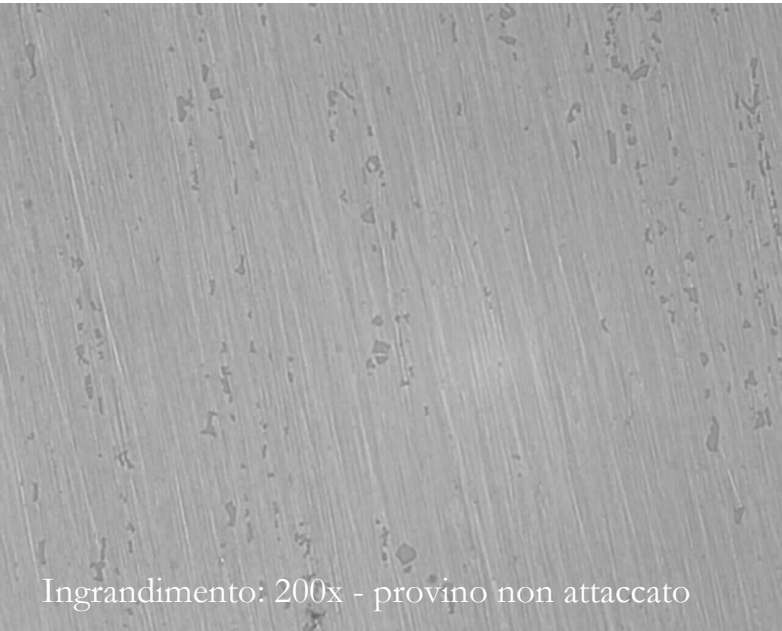

Figura 31: Provino centrale attacco 2.

Figure 31: Central specimen, attachment 2.

A titolo di confronto, è mostrata la visualizzazione cromatica delle seconde fasi di colore scuro su un provino ricavato dall'attacco A e su un provino ricavato dall'attacco 2, Fig. 32. Si nota nel caso dell'attacco rotto un allineamento continuo ed ordinato delle seconde fasi significativamente più pronunciato che nel caso dell'attacco di confronto.

Riassumendo:

Tutti i campioni metallografici indagati confermano il verso di laminazione previsto dalla specifica di progetto.

L'analisi microstrutturale eseguita sui campioni ricavati dagli attacchi A e D, analoghi tra loro, mostra sostanziali differenze con quanto individuabile sui campioni prelevati dagli attacchi 1 e 2 , sintetizzabili nel modo seguente:

- maggiore stratificazione dei precipitati grigio chiari lungo i bordi longitudinale dei grani;

- $\quad$ precipitati di colore grigio scuro e nero visibili a basso ingrandimento;

- pori e cavità a morfologia dendritica, correlabili a fenomeni di overheating/eutectic melting, cerchi rossi sull'immagine tratta dall'atlante metallografico in Fig. 33;

- precipitati globulari a struttura dendritica, anche'essi correlabili a fenomeni di overheating/eutectic melting (rosettes), frecce verdi sull'immagine tratta dall'atlante metallografico in Fig. 31. 

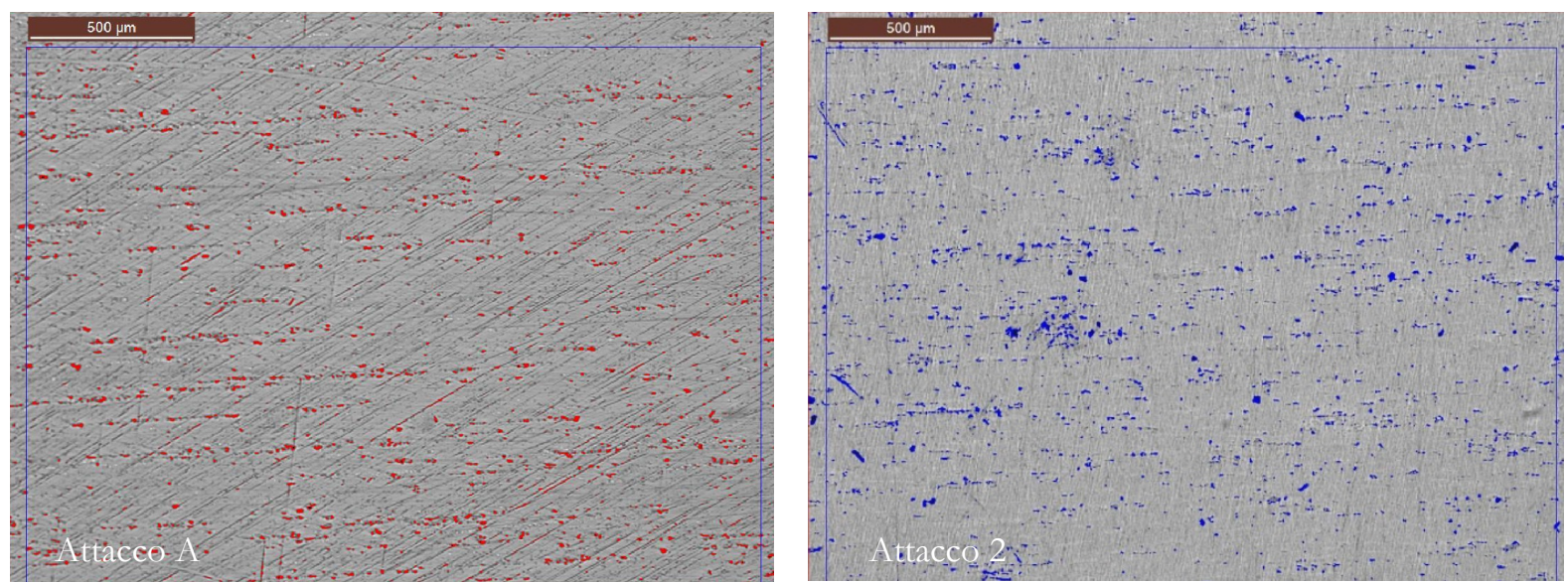

Figura 32: Confronto delle seconde fasi.

Figure 32: Different boundary grain precipitates (second phases).

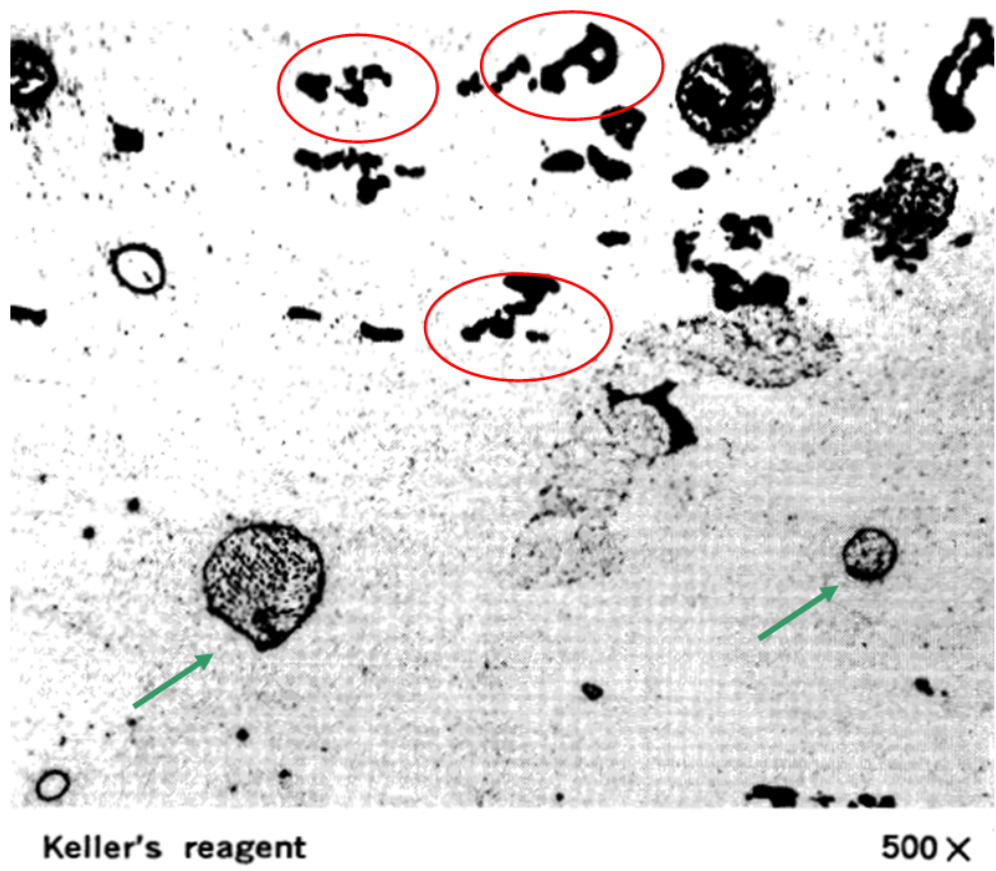

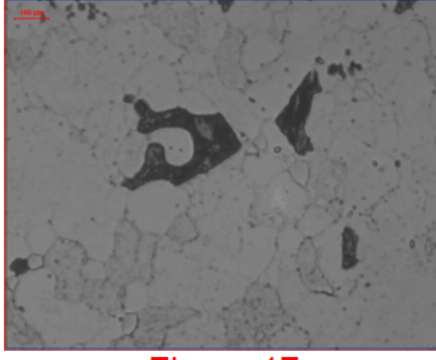

Figura 17

Figura 20

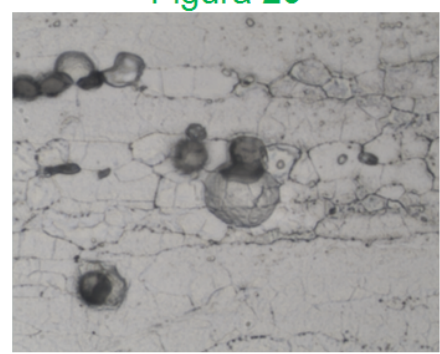

Figura 33: AA2014 T6, cavità dendritiche e rosettes dovute a overheating, da manuale[1].

Figure 33: AA2014 T6, dendritic cavities and rosettes due to overheating/eutectic melting phenomena [1].

Analisi delle seconde fasi

Sulle seconde fasi già identificate mediante esame FESEM ed esame metallografico e sulle particelle globulari a morfologia dendritica individuate sulla superficie di rottura, è stata condotta l'analisi FESEM-EDS finalizzata ad identificarne la composizione.

Nella composizione delle seconde fasi non si rinvengono elementi diversi da quelli prevedibili nella lega AA2014. Si evidenzia concentrazione degli elementi Magnesio e Silicio sui precipitati scuri (associabili a $\mathrm{CuMg}_{5} \mathrm{Si}_{4} \mathrm{Al}_{4}$ e $\mathrm{MgSi}_{2}$ ), $\mathrm{Rame}$ sui precipitati grigi chiari (associabili a $\mathrm{CuAl}_{2}$ ), Manganese e Silicio e Ferro sui precipitati grigi scuri (associabili a $\left.(\mathrm{Fe}, \mathrm{Mn})_{3} \mathrm{SiAl}_{12}\right)$, Fig. 34.

Anche i globuli a struttura dendritica risultano costituiti dagli elementi di lega AA 2014, tuttavia vi si concentra l'elemento Ferro, Fig. 35 . 


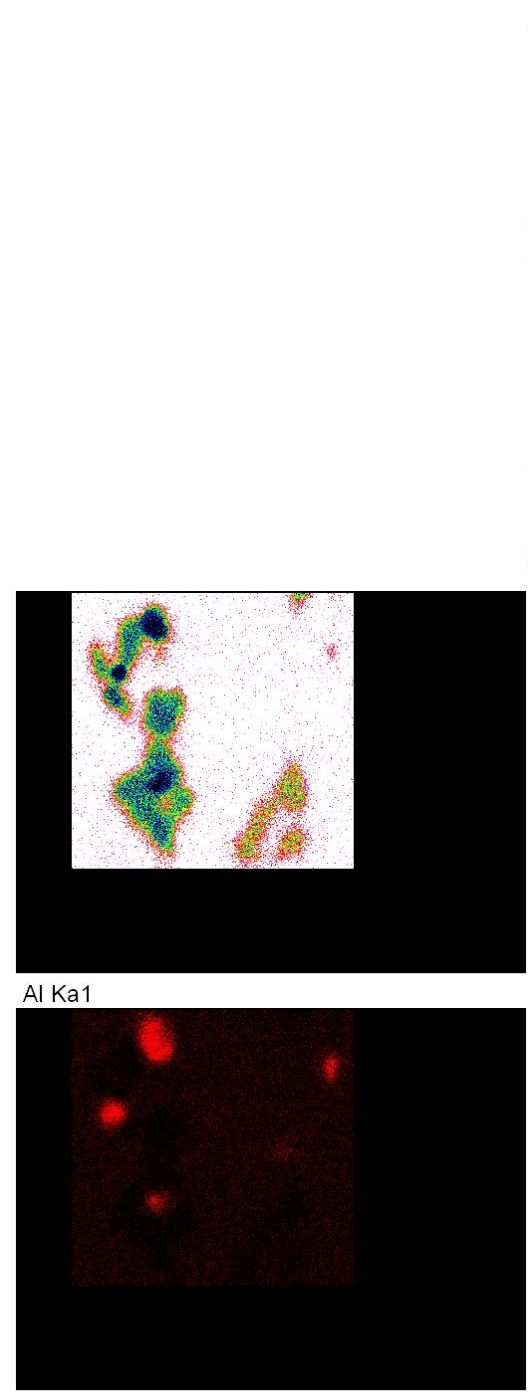

Mg Ka1_2
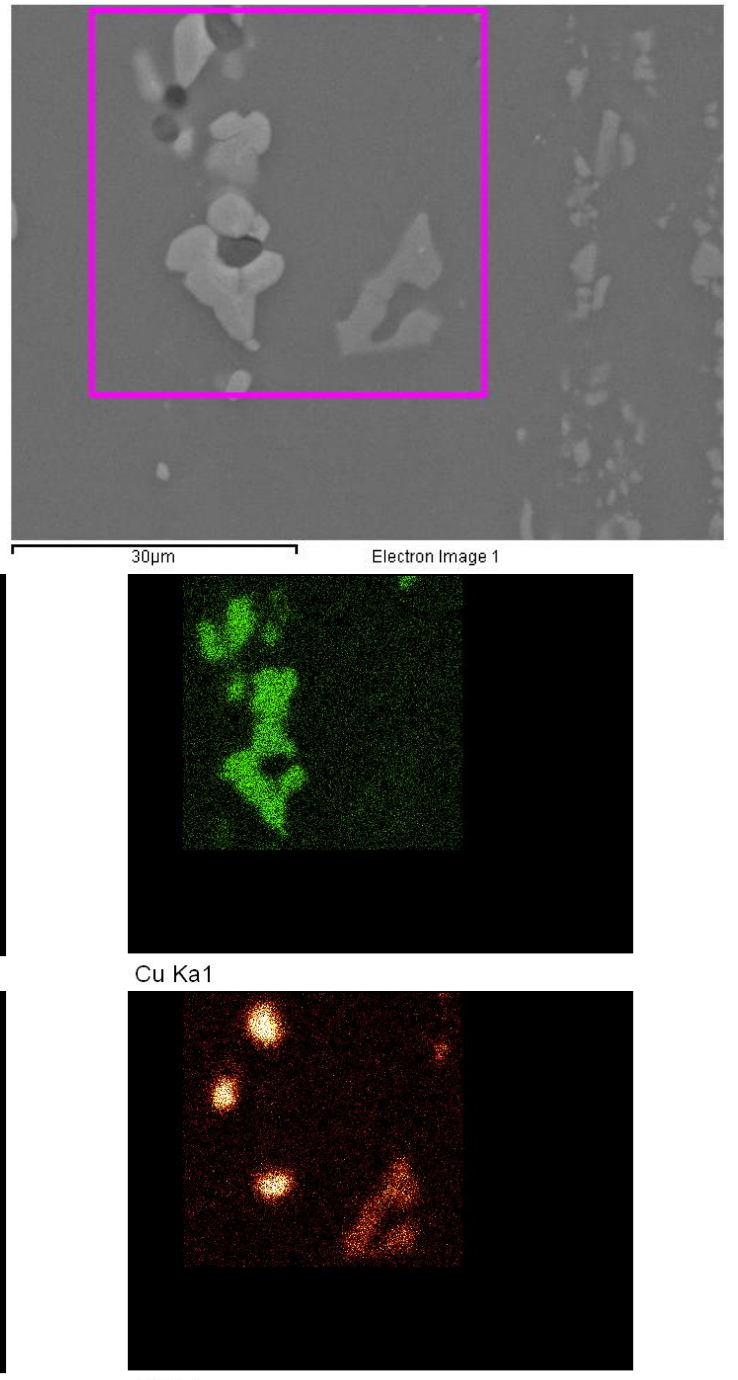

Si Ka1

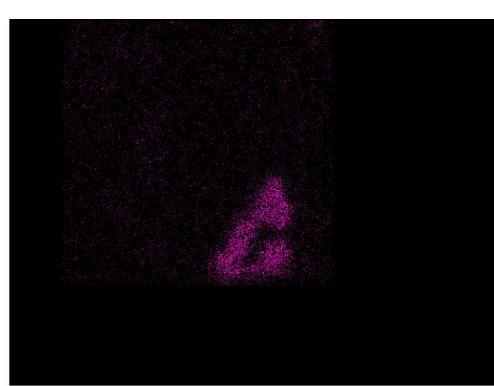

Fe Ka1

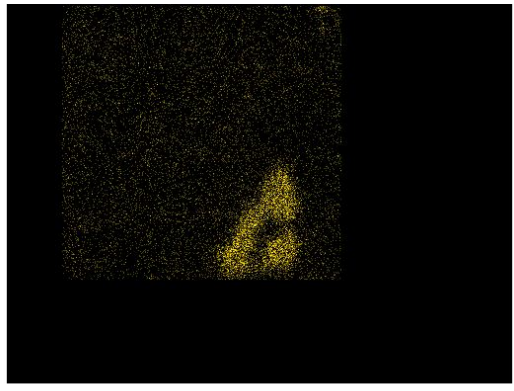

Mn Ka1

Figura 34: Analisi elementare delle seconde fasi.

Figure 34: Elemental analysis (EDS) of second phases.
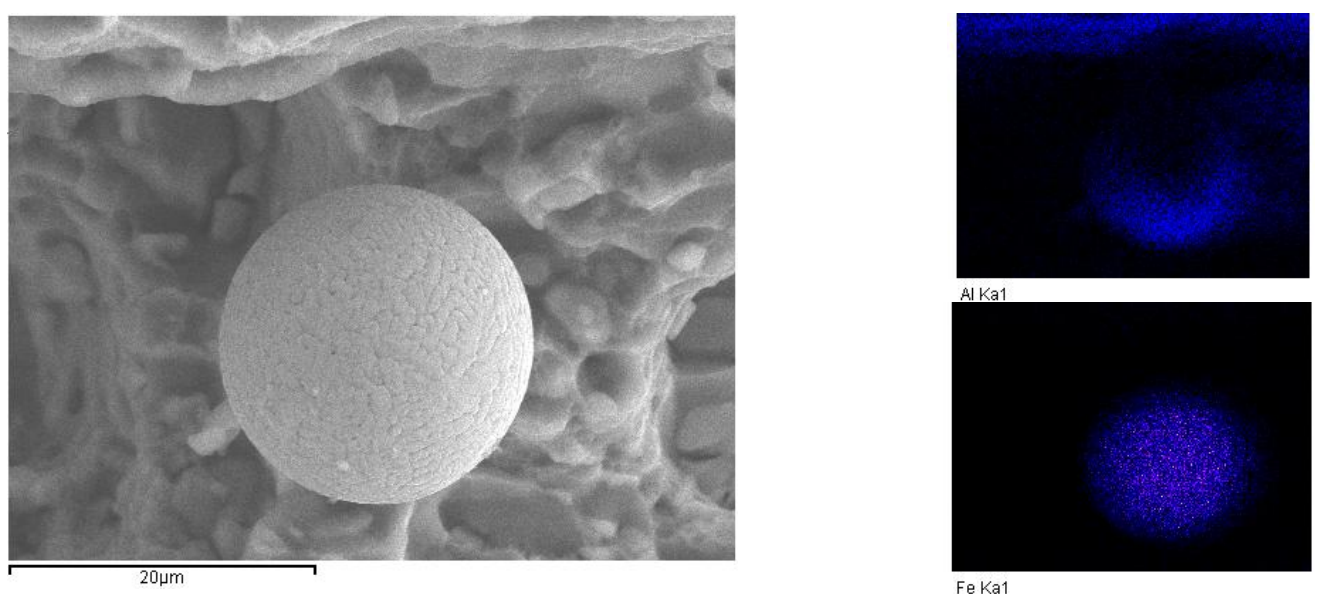

Figura 35: Analisi elementare di globulo a morfologia dendritica.

Figure 35: Elemental analysis (EDS) of a globular dendritic formation. 
Valutazione della resistenza alla corrosione intergranulare

Campioni prelevati dagli attacchi A, D, 1 e 2 sono stati sottoposti alla prova standardizzata di promozione della corrosione intergranulare [2], al fine di valutarne il grado di severità.

I provini metallografici, ricavati da sezioni dei campioni in punti che hanno sofferto il maggiore attacco superficiale, indicano che i campioni prelevati dagli attacchi A e D sono maggiormente suscettibili alla propagazione della corrosione intergranulare rispetto all'attacco 2 (in termini di profondità ed estensione dell'attacco corrosivo) e all'attacco 1 (in termini di numerosità delle ulcere e dei fenomeni corrosivi) Fig. 36.
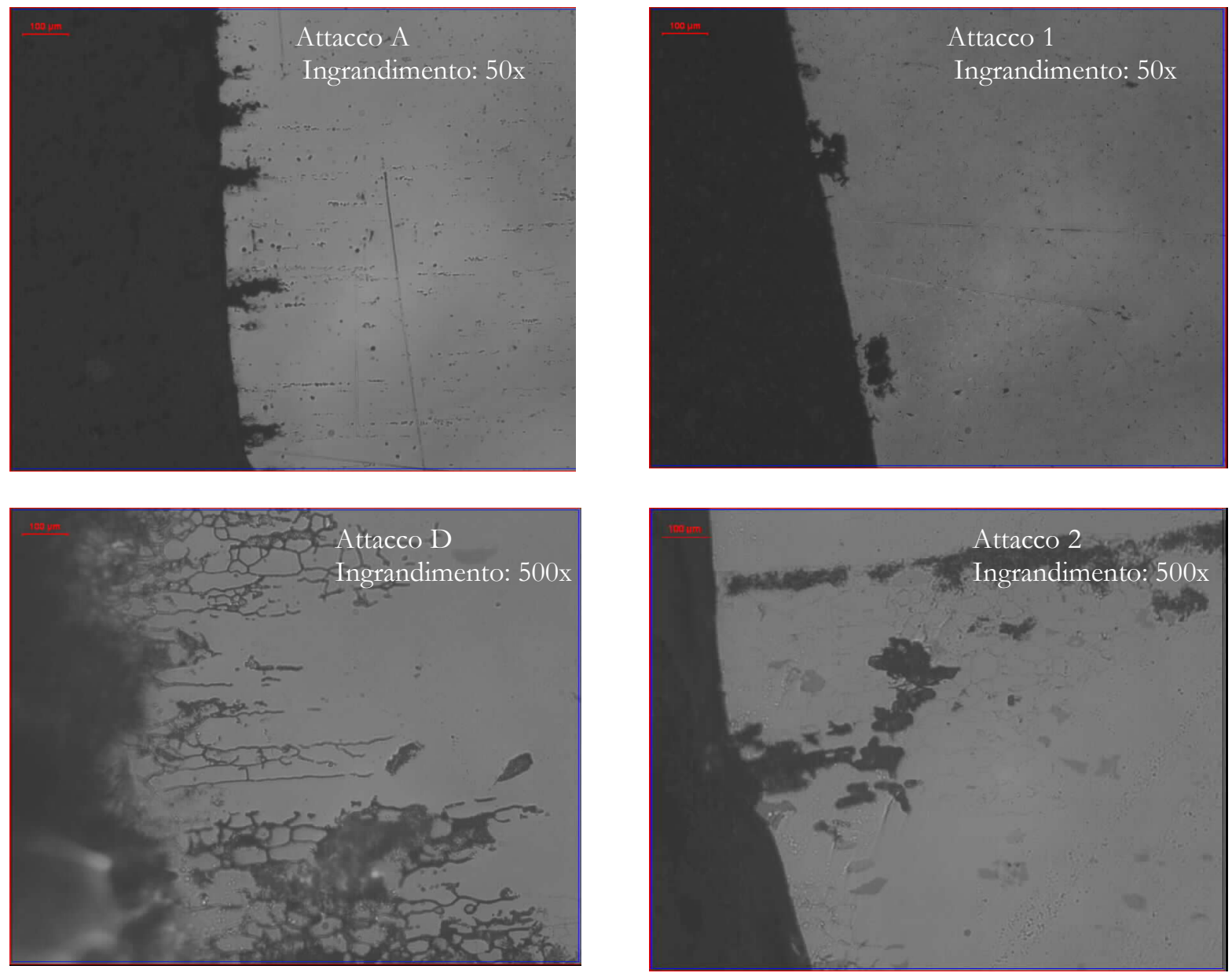

Figura 36: Resistenza a corrosione intergranulare.

Figure 36: Intergranular corrosion resistance.

\section{Determinazione di durezza}

Le misure di durezza sono state effettuate secondo il metodo Rockwell $\mathrm{T}$ con un carico di $30 \mathrm{~kg}$ e penetratore sferico di acciaio Ø 1/16" su sezioni dell'attacco S/N 4468 prodotte sul lato longitudinale e sul lato corto dei grani.

\begin{tabular}{ll}
\hline Ubicazione misura & Durezza \\
Sezione longitudinale & $71 \mathrm{HRT}$ \\
Sezione corta & $69 \mathrm{HRT}$ \\
\hline
\end{tabular}

Tabella 5: Valori di durezza rilevati sull'attacco A.

Table 5: Hardness values as measured on the failed attachment.

Il valore medio di durezza pari a 71 HRT è corrispondente ad un valore di $133 \mathrm{HRB}$ ed a un carico unitario di rottura dedotto di $52 \mathrm{Kg} / \mathrm{mm}^{2}$ e risulta regolare per una lega a base alluminio del tipo AA2014 nello stato di trattamento termico T651 [3]. 


\section{Analisi agli elementi finiti}

Il calcolo mediante simulazione numerica (76.000 elementi tetraedrici a 4 nodi) è stato effettuato con un' analisi lineare elastica e con l'introduzione di algoritmi di contatto tra le superfici dei bulloni e gli occhielli del componente allo scopo di simulare il più fedelmente possibile lo stato di sollecitazione locale. Ciò ha permesso di effettuare un'analisi strutturale statica finalizzata alla determinazione degli stress agenti sull'attacco A nelle normali condizioni di esercizio, come di seguito stimato:

$\checkmark$ pressione dell'impianto idraulico pari a $1500 \mathrm{psi}$;

$\checkmark$ materiale del bullone: acciaio ad alta resistenza;

$\checkmark \quad$ forza di serraggio dei dadi sui prigionieri pari a $20 \mathrm{MPa}$.

La simulazione ha permesso di individuare la zona di massima sollecitazione del componente, Fig. 37, ed il valore di stress principale massimo pari a circa $20 \mathrm{MPa}$ nella zona dei bordi esterni della superficie di tipo 1, Fig. 38.

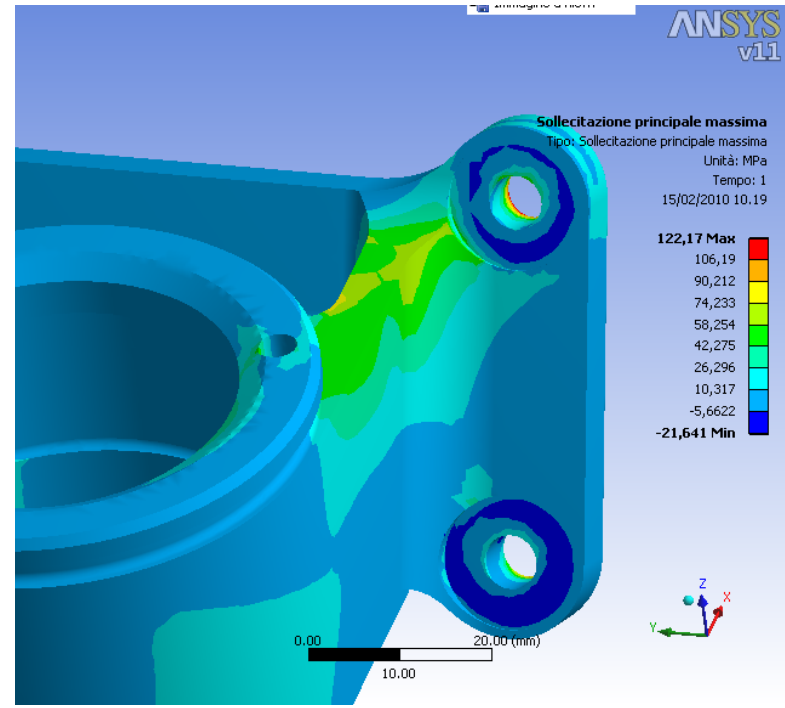

Figura 37: Simulazione della sollecitazione. Figure 37: Stress simulation.

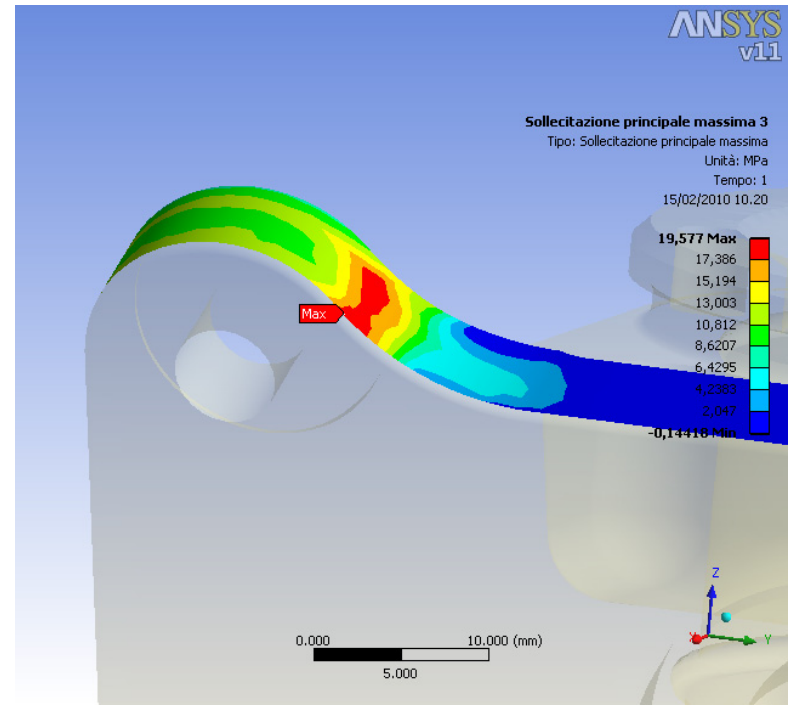

Figura 38: Stress principale. Figure 38: Principal stress.

Tale stress comporta tensione perpendicolare all'asse di laminazione riscontrato in esito agli esami microstrutturali, Fig. 39.

L'analisi della deformata indica la direzione principale della deformazione, ortogonale al piano sul quale giace lo stress della zona d'innesco, freccia bianca in Fig. 40.

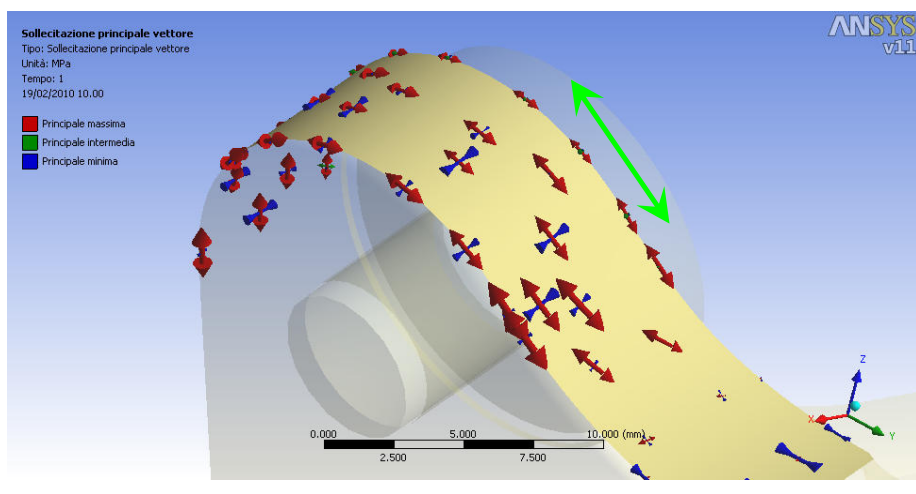

Figura 39: Tensione determinata dallo stress in Fig. 38. Figure 39: Tensile stress due to the stress of Figure 38.

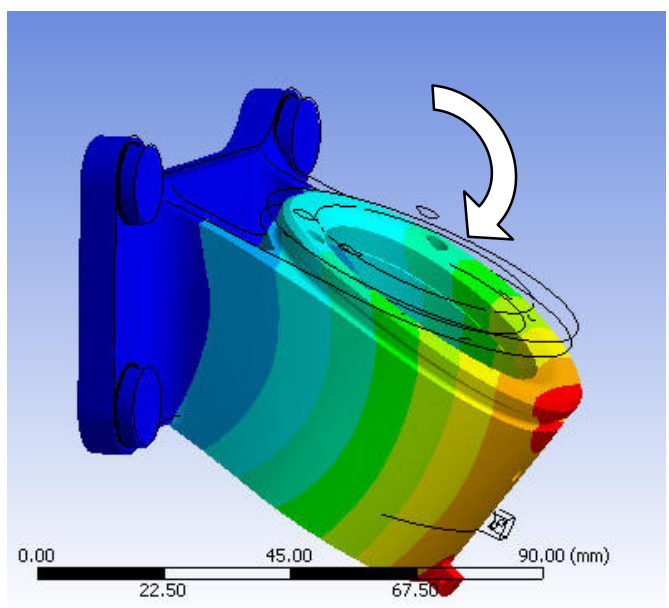

Figura 40: Deformata. Figure 40: Strain principal direction. 


\section{CONCLUSIONI}

$\mathrm{L}$ e rotture rinvenute sull'attacco sono simmetriche per posizione, morfologia e piani di propagazione, pur avendo questi estensioni differenti. Sono pertanto associabili allo stesso meccanismo di rottura.

Ad escludere che la rottura sia l'effetto dell'urto del rotore principale con il suolo è proprio il carattere simmetrico delle rotture osservate rispetto alla direttrice coda-prua del velivolo.

Le forme di propagazione della rottura mostrano in microscopia elettronica le stesse caratteristiche di propagazione intergranulare con presenza di micropori al bordo dei grani e modica quantità di prodotti di corrosione.

Anche l'esame metallografico della sezione operata sulla superficie di rottura conferma per tali forme il meccanismo di rottura intergranulare, con molteplici ramificazioni di modesta profondità.

Le zone in prossimità del foro al bordo superiore sono risultate essere alla simulazione, le più esposte a stress di natura tensionale nelle normali condizioni di esercizio e, sebbene la presenza di segni di martellamento non consenta l'individuazione di chiari punti di innesco, la maggiore quantità di prodotti di corrosione che vi si rinviene le individua quali aree di inizio della rottura progressiva.

L'orientazione dei micropori sulle superfici di tipo 3 ed i segni di battitura sulle superfici di tipo 1 sono compatibili con un carico tensionale con componente ortogonale alla parte dell'attacco costituente la piastra di fissaggio e con il conseguente distacco del frammento centrale dell'attacco per avanzamento lungo la direttrice coda-prua del velivolo.

I segni morfologici individuati sulla superficie di rottura e sulla sua sezione metallografica sono tutti compatibili con un meccanismo di tensocorrosione $[4,5]$.

Le caratteristiche morfologiche di fragilità della superficie trovano spiegazione in quanto osservato a livello micro strutturale ed in termini di contenuto di idrogeno.

L'analisi metallografica eseguita sui campioni estratti dagli attacchi A e D, di microstruttura analoga, indica che in essi le seconde fasi sono stratificate maggiormente rispetto ai provini ottenuti da lotti di produzione differenti.

Tali fasi, presenti nelle specie attese, sono infatti allineate ai bordi dei grani, con formazione grossolana dei precipitati a base di Ferro, Manganese e Silicio.

Il contenuto di idrogeno misurato su campioni ricavati dall'attacco A è superiore di un ordine di grandezza rispetto a quanto riportato nel certificato di produzione ed è tre volte superiore a quello misurato per gli altri attacchi.

La bibliografia riporta che il materiale AA2014 viene indurito per precipitazione, principalmente della specie $\mathrm{CuAl}_{2}$. Questo precipitato è atteso non essere particolarmente fine, poiché la sua completa solubilizzazione nella lega è impedita dal limite di temperatura da non superare per non incorrere nella fusione dei complessi a base di Magnesio e Silicio.

La necessità di disperdere al massimo la fase ricca in Rame comporta dunque che l'intervallo termico di soluzione solida ha il limite massimo $\left(507^{\circ} \mathrm{C}\right)$ molto vicino al valore termico cui inizia la fusione eutettica $\left(510{ }^{\circ} \mathrm{C}\right)[6]$.

Fin dagli anni 60, la lega AA2014 è riconosciuta essere suscettibile a tensocorrosione. Tale predisposizione è principalmente associata alla seconda fase a base di $\mathrm{CuAl}_{2}[7]$, quando essa è presente in agglomerati grossolani o allineati. Tale disposizione si realizza per surriscaldamento oltre $507^{\circ} \mathrm{C}$, o per raffreddamento eseguito troppo lentamente.

L'allineamento anormale di precipitati grossolani a base di Ferro nel verso di laminazione, che può rinvenirsi anche in una sola parte dell'estruso di AA 2014 T651, causa riduzione delle proprietà meccaniche e tendenza alla rottura fragile nella direzione perpendicolare (short transverse direction) [8].

Inoltre, la presenza d'idrogeno nelle leghe di alluminio induce essenzialmente infragilimento [9]ed agisce quale promotore del meccanismo di rottura intergranulare, poiché si addensa presso le seconde fasi di bordo grano[10].

$\mathrm{Nel}$ caso osservato, la microstruttura stratificata con le seconde fasi allineate al bordo longitudinale dei grani, le cavità dendritiche e le strutture dendritiche individuate lasciano supporre che i suddetti attacchi provengano da un lotto di materiale che possa aver sofferto fenomeni di surriscaldamento e/o fusione eutettica. Tale supposizione è suffragata dalla maggiore suscettibilità degli attacchi $\mathrm{A} \mathrm{e} \mathrm{D}$ alla prova di resistenza a corrosione intergranulare.

Le rinvenute alterazioni microstrutturali, la suscettibilità a corrosione intergranulare e l'eccessivo contenuto di idrogeno potrebbero avere agito quali uniche driving forces del meccanismo di corrosione tensoassistita.

In merito alla conformità a specifica dei particolari in uscita dal processo produttivo è possibile ipotizzare quanto segue. $\mathrm{Si}$ suppone che attacchi provenienti dallo stesso lotto di produzione abbiano le stesse caratteristiche poiché ben 50 attacchi vengono ricavati per lavorazione meccanica della stessa barra di materiale AA2014 prodotta per estrusione e sottoposta a trattamento termico T6511. Un errore nell'effettuazione del trattamento termico avrebbe effetto su tutta la barra, ma non necessariamente nello stesso modo e con la stessa gravità su tutti i 50 attacchi ottenibili dalla stessa.

La conformità a specifica del materiale in uscita dalla fabbrica prevede, per lo stato termico T651, le seguenti prove di verifica per le proprietà meccaniche: prova di trazione, prova di durezza, prova di conduttività elettrica, in base alle quali, 
almeno nel caso da noi affrontato, nulla di anomalo sarebbe risultato. Non sono previsti invece l'esame metallografico né alcun test di resistenza a corrosione intergranulare che porterebbero ad evidenziare anomalie di questo genere e che potrebbero essere effettuati su scarti di lavorazione.

\section{BIBLIOGRAFIA}

[1] ASM Committee on Metallography of Aluminum Alloys, Microstructure of Aluminum Alloys in Metals Handbook, $8^{\text {th }}$ Edition, American Society for Metals, Ohio, 7 (1972).

[2] ASTM G 110-92, Standard practice for Evaluating Intergranular Corrosion Resistance of Heat Treatable Aluminium Alloys by Immersion in Sodium Chloride + Hydrogen Peroxide Solution, ASTM International, West Conshohocken PA, (2003).

[3] ASM International Committee, Fabrication and Finishing of Aluminum Alloys - Heat Treating, in ASM Specialty Handbook Aluminum and Aluminum Alloys, 4th ed., J.R. Davis, Ohio, (1998) 290-327.

[4] Shreir, L.L., Jarman, R.A., Burstein, G.T., Corrosion, 3 ${ }^{\text {rd }}$ ed., Ed. BH, Oxford, 1 (2000).

[5] Russel, H.J., Stress Corrosion Cracking of Aluminum Alloys, In: Stress corrosion cracking - Material performance and evaluation, Russel H.J., ASM International, Ohio, (1993).

[6] ASM International Handbook Committee, Heath Treating of Aluminum Alloys, in ASM Handbook, 2 ${ }^{\text {nd }}$ ed., ASM International, Ohio, 4 (1997) 841-879.

[7] Bell AeroSystems, Fusion Welding of 2014 Aluminum Alloy, HLR 61-41 M rev A (1962).

[8] Arieli, A., Gilad, Y., Blas, A., Investigation of the microstructure uniformity and short transverse mechanical properties of the AL2014 T651 plates, SAMPE Quarterly, (1980) 16-21.

[9] Speidel, M.O., Hydrogen Embrittlement and Stress Corrosion Cracking of Aluminum Alloys, in Gibala, R., Hehemann, R.F., Hydrogen Embrittlement and Stress Corrosion Cracking, American Society for Metals, Ohio, (1984) 271-296.

[10] Ambat, R., Dwarakadasa, E.S., Effect of Hydrogen in aluminium and aluminium alloys: a review, Bull. Mater. Sci., 19(1) (1996) 103-114. 